\title{
On the Smoothness of Value Functions and the Existence of Optimal Strategies in Diffusion Models
}

\author{
Bruno Strulovici* $\quad$ Martin Szydlowski ${ }^{\dagger}$
}

March 26, 2015

\begin{abstract}
Studies of dynamic economic models often rely on each agent having a smooth value function and a well-defined optimal strategy. For time-homogeneous optimal control problems with a one-dimensional diffusion, we prove that the corresponding value function must be twice continuously differentiable under Lipschitz, growth, and non-vanishing-volatility conditions. Under similar conditions, the value function of any optimal stopping problem is shown to be (once) continuously differentiable. We also provide sufficient conditions, based on comparative statics and differential methods, for the existence of an optimal control in the sense of strong solutions. The results are applied to growth, experimentation, and dynamic contracting settings.
\end{abstract}

Keywords: Optimal Control, Optimal Stopping, Smooth Pasting, Super Contact, Strong Solution, Comparative Statics, Bounded Variation, Markov Control, HJB Equation.

JEL codes: C61, C73, D86, O40.

\section{Introduction}

Ever since Louis Bachelier formalized the concept of Brownian Motion to study financial markets, diffusion processes have played an increasingly important role in economic analysis. Diffusions are routinely used to model macroeconomic and financial shocks, news arrival in learning and experimentation settings, stochastic output and demand, as well as many other uncertain processes. Harnessing the relevant techniques has proved a challenge to economists, however, so much so that it has motivated a book by Dixit (1993) on the "Art of Smooth Pasting," which included a heuristic justification for the differentiability of value functions at optimal stopping thresholds.

In dynamic models, the smoothness of value functions is often necessary to investigate the properties of optimal decisions and payoffs, as illustrated by the celebrated Benveniste-Scheinkman theorem.

\footnotetext{
${ }^{*}$ Corresponding author. Department of Economics, Northwestern University, 2001 Sheridan Road, Evanston, Illinois 60208, USA. E-mail Address: b-strulovici@northwestern.edu. Strulovici gratefully acknowledges financial support from an NSF CAREER Award (Grant No. 1151410) and a fellowship form the Alfred P. Sloan Foundation.

${ }^{\dagger}$ Department of Finance, Carlson School of Management, University of Minnesota, 321 19th Ave South, Minneapolis, MN 55455, USA. E-mail Address: szydl002@umn.edu.
} 
In growth models, for instance, it is used together with the Bellman - or, in continuous-time, the Hamilton-Jacobi-Bellman "HJB" - equation to establish show that an agent's optimal consumption and investment decisions are monotonic in her wealth and other parameters of the model. In dynamic contracting models, the agent's optimal effort and consumption, his continuation utility, and the impact of the principal's risk attitude are typically analyzed via the HJB equation, even though the equation typically has no closed-form solution. In bandit problems, many properties of the optimal experimentation strategy can be similarly characterized without an explicit solution, as we also illustrate.

In these and other applications, one needs to establish, before proceeding to any further analysis, that the value function is smooth enough to solve the HJB equation 11 For the optimal control of diffusions, "smoothness" thus requires (and means in this paper) twice differentiability of the value function, and is sometimes called the super contact condition $\mathrm{L}^{2}$ In pure stopping problems, "smoothness" requires (and means) that the value function is once differentiable, and is known as the smooth pasting property.

Unfortunately, economists do not have available at hand a simple theorem guaranteeing the appropriate level of smoothness for the value functions arising in their diffusion models. "Verification theorems" are used to check that a smooth candidate solution is equal to the value function, but they neither provide this candidate solution, nor do they guarantee its existence. In optimal stopping problems, the usual arguments for smooth pasting are heuristic, and they typically assume that the optimal stopping region takes on a very specific "threshold" form. In optimal control problems, economists have had to rely on ad hoc arguments to establish that the value functions of their particular models solved the HJB equation.

This paper provides two theorems for the smoothness of value functions, based on simple and readily checked conditions. These theorems pertain to infinite-horizon models, ubiquitous in economics, in which the state is a one-dimensional, time-homogeneous diffusion. The first theorem states - under continuity, linearity, and non-vanishing-volatility conditions - that the value function of any optimal control problem is twice continuously differentiable and solves everywhere the HJB equation corresponding to that problem. The theorem equally applies to bounded and unbounded state spaces, and requires only that the control lie in a compact set. In particular, the control space need not be convex or one dimensional.

\footnotetext{
${ }^{1}$ Unless stated otherwise, solutions should be understood in a "classical" sense. To exploit the HJB equation and derive properties of value functions and optimizers, classical solutions are significantly simpler and easier to work with than other solution concepts.

${ }^{2}$ The super contact condition has been extensively used to characterize optimal solutions. See, e.g., DeMarzo and Sannikov (2006) and DeMarzo et al. (2012).
} 
This theorem relaxes several assumptions, problematic in economic applications, made in the large body of work dealing with the existence of solutions to boundary value problems, which include HJB equations. For example, Krylov (1980, Th. 5, p. 24) assumes a bounded state space and a convex control space, which respectively rules out growth models and bandit problems; Noussair (1979), Safonov (1989), and Gilbarg and Trudinger (2001) assume that the coefficients of the diffusion are Hölder continuous in the state, a property that is obviously violated when the conrol space is discrete ${ }^{3}$ Fleming and Soner (1993, p. 161) assume a bounded domain and bounded coefficients with continuous derivatives in the state 4

We focus on the existence of twice differentiable (or "classical") solutions because many methods to obtain qualitative properties of the value function (which in turn determine properties of the optimal action and the state trajectory) rely on twice differentiability $\left.\right|^{5}$ More general solution concepts, such as weak ${ }^{6}$ or viscosity $\sqrt{7}$ solutions, do not guarantee the existence of a second derivative. This makes it difficult to qualitatively characterize the value function and to use a first-order condition to derive the optimal control in terms of its derivatives, since these may not exist.

Our second smoothness theorem states, under the same conditions, that the value function of any stopping problem is continuously differentiable, as long as the terminal value function has this property 8 For both theorems, the conditions were previously known to be necessary for the results $9^{9}$ We are not aware of previous theorems showing that these conditions, taken together, are sufficient.

Just because the value function of a control problem is smooth, this does not imply the existence of an optimal control for that problem - not even when the HJB equation has a maximizer at every state. The issue is whether the candidate control coming from the HJB equation generates a welldefined trajectory for the state process. Establishing the existence of an optimal control is of clear interest in many settings. For instance, it may be used to show the existence of a best-response

\footnotetext{
${ }^{3}$ These results, unlike ours, apply to multidimensional optimization, which explains some of the more stringent conditions that they assume.

${ }^{4}$ They relax the bounded-coefficients assumption on p. 163, at the cost of assuming a more specific functional form for the drift coefficient. Their method applies to multidimensional control problems with time-dependent coefficients.

${ }^{5}$ See, e.g., the concavity arguments in Sannikov (2008) and DeMarzo and Sannikov (2006) and the qualitative characterization obtained by Shreve et al. (1984).

${ }^{6}$ See e.g. Krylov (1980), Ch. 4.

${ }^{7}$ See Crandall and Lions (1983) and Lions (1983).

${ }^{8}$ The arguments can be extended to combined control and stopping problems. See Section 5

${ }^{9}$ Touzi (2010) provides an example with an unbounded control space in which the value function is not differentiable. Øksendal and Sulem (2007, p.139) consider a pure stopping problem in which the terminal function is not differentiable, and show that the value function is not differentiable either at one of the stopping thresholds. With a vanishing volatility, the smoothing effect of the Brownian motion is absent at some states, and it is easy to build deterministic examples in which the value function is not twice differentiable.
} 
and, subsequently, of an equilibrium, in continuous-time games, or in arguments using an optimal control to derive properties of the value function. Most obviously, existence is also required to derive properties of the optimal control itself.

Our second contribution is to provide sufficient conditions for the existence of an optimal control in the problems described above. There are two concepts of solutions to stochastic differential equations: The strong one requires that the state trajectory be entirely determined by the control process and by the realization of exogenous uncertainty. This solution corresponds to one's intuition based on discrete-time stochastic optimization. The weak solution concept requires only that there exist some probability space for which the state process and Brownian Motion satisfy the state equation. Here, the Brownian Motion is determined as part of the solution, and the state trajectory need not be determined by the realized path of the Brownian Motion. While weak solutions are easily shown to exist 10 they are of limited interest when it comes to economic applications. Indeed, while the vast majority of economic models specify the source of uncertainty explicitly, ruling out the possibility that it might be freely chosen, the weak solution concept supposes that the structure of uncertainty is endogenous to the solution. Moreover, even when the control is a function of the current state, i.e. Markovian, the trajectory of a weak solution is not pinned down by this function and the realized path of the Brownian Motion used to model exogenous uncertainty. This makes the discrete-time intuition and interpretation of a controlled state inapplicable, in which the agent reacts to exogenous shocks and generates the state trajectory. Allowing weak solutions thus introduces conceptual differences between continuous-time and discrete-time models which are not present under strong solutions.

In economic models with a controlled diffusion, there are currently few options, other than finding an explicit solution, for guaranteeing the strong existence of an optimal control 11 This difficulty can be easily seen in any control problem where the control set is binary, such as a two-armed bandit problem. In these problems, any nontrivial Markovian control has to "jump" as the diffusion hits some regions of the state space, creating discontinuities in the coefficients generating the stochastic equation for the state. This destroys the Lipschitz continuity typically required for the existence of a solution; in these applications, the coefficient is not even continuous.

Fortunately, there exist specific results for the case of one-dimensional diffusions, which guarantee

\footnotetext{
${ }^{10}$ Measurability and local integrability of the controlled drift and non-degeneracy and local integrability of the squared inverse of the controlled volatility suffice to show such existence. See e.g. Karatzas and Shreve (1998), p. 339 .

${ }^{11}$ Stroock and Varadhan (1979), Ch. 6 and 7 and Krylov (1980), Th. 1, p. 87, developed results showing the existence of weak solutions. For more general existence results with weak solutions, see the survey by Borkar (2005). It is also well known, and shown in this paper (Section 3), that there always exist $\varepsilon$-optimal controls that generate strong solutions.
} 
the existence of a unique strong solution as long as the volatility coefficient has bounded variation 12 Building on these results, we identify conditions for the existence of an optimal control. These conditions are all based on the HJB equation of the control problem, exploiting the fact that the value function indeed solves that equation. Proving the existence of an optimal strategy thus provides a first application of our smoothness results.

Some of our existence results are based on establishing, via an envelope theorem, differentiability of the control, and are illustrated in several applications. Our other existence results are based on the theory of monotone comparative statics: if one can show that the control constructed from the HJB equation is monotonic, or at least piecewise monotonic, this will guarantee that it has bounded variation and, under a simple regularity condition, that the volatility has bounded variation. Proving such monotonicity is nontrivial, because it is typically impossible to explicitly compute the value function and its derivatives. However, it is precisely for this kind of situations that the theory of comparative statics has been developed.

Our results are illustrated with several applications. The first one concerns an optimal growth problem. Relaxing usual concavity and convexity assumptions, we show that the value function is always smooth and solves the Bellman equation under standard regularity conditions. We also discuss conditions for the existence of an optimal control. Our second application concerns multiarmed bandit problems with a common state. Each arm is a choice that is informative about the true state of the world, and has a state-dependent payoff. The value function is, without any further assumptions, always twice differentiable. When the number of arms is finite, the number of switching points between arms is shown to be finite (and uniformly bounded), although it may be strictly optimal to use a given arm over disjoint intervals of beliefs. We also provide general conditions for the existence of an optimal control when the agent can also allocate resources across two arms, rather than merely choose one of them. The third application revisits the principalagent model analyzed by Sannikov (2008), proving the existence of an optimal contract under a simple condition on the effort cost function 13 We also provide, as a simple illustration of our results, a general proof that the option value of waiting in optimal stopping problems is positive and increasing in the volatility of the underlying process.

\footnotetext{
${ }^{12}$ The first such result is due to Nakao (1972). See Section 4

${ }^{13}$ Without this condition, the continuation utility process derived for the contract characterized in that paper is only guaranteed to exist in the sense of a weak solution.
} 


\subsection{Literature Review}

In addition to the papers already mentioned, twice differentiability in optimal control problems has been studied by Evans (1983), who assumes a finite control space, and for specific models in Operations Research (see, e.g., Harrison and Taksar (1983)). A variety of methods have been used to establish that property. Krylov (1980), Chapter 4, relies on probabilistic methods and Gilbarg and Trudinger (1980) and Safonov (1989) rely on fixed point arguments. By contrast, the present results are obtained via the shooting method and are based on the insights of Schrader (1969). They can easily be extended, for example to include vanishing volatility as in the extension of Appendix D or to include boundary conditions involving the derivative, as in Szydlowski (2014). Similar results have been obtained via less elementary methods by De Coster and Habets (2006), Th. 1.3, p. 77, Hartman (1960), and, for the case of bounded domains, Bebernes (1963). As in the present paper, De Coster and Habets exploit Nagumo's condition to prove their smoothness result. They assume the existence of so-called lower and upper solutions to the boundary value problem. Our method delivers upper and lower solutions as a byproduct and we do not need to assume their existence a priori.

Recent results on the necessity of smooth pasting are provided by Dayanik and Karatzas (2003), Peškir and Shiryaev (2006), Chapter 9.1, and Villeneuve (2007), using methods based on the scale function of the SDE, or by "excessivity" of the value function. These results focus on settings without flow payoffs. The integral of the flow payoff may be converted into an additional state. Unfortunately, this conversion creates a multidimensional state space, in which some states have zero volatility. Peškir and Shiryaev (2006) propose a method for proving the necessity of smooth pasting which, they mention, can be extended to multidimensional states. However, that method requires a minimal volatility condition that is not satisfied by the converted state. Pham (2009, Prop 5.2.1) contains a result similar to ours based on viscosity solutions, but does not spell out the conditions on the primitives under which the viscosity solution has the Lipschitz property used in his analysis.

Fleming and Soner (1993), p. 159, prove the existence of a strong solution for controlled SDE problems and assume a priori that the control is Lipschitz in the state (and jointly continuous in the time-state space, when it also depends explicitly on time). Yong and Zhou (1990), Th. 5.2, p. 68, prove a similar result for linear controlled SDEs assuming a convex action set. The present paper generalizes both results.

The paper is organized as follows. Section 2 introduces the general optimal control problem. Section 3 proves that the value function is twice continuously differentiable. Section 4 provides sufficient conditions for the existence of an optimal control. Section 5 turns to the optimal stopping prob- 
lem, proving the continuous differentiability of the value function. Sections 35 include examples illustrating their respective results. Section 6 concludes. Technical proofs are in the Appendix.

\section{Control Problem}

We are given a filtered probability space $\left(\Omega, \mathcal{F},\left\{\mathcal{F}_{t}\right\}_{t \in \mathbb{R}_{+}}, \mathbb{P}\right)$ which satisfies the usual conditions and whose outcomes are identified with the paths of a standard Brownian motion, denoted by $B{ }^{14}$ We consider a process $\left\{X_{t}\right\}_{t \in \mathbb{R}_{+}}$controlled by another process $\left\{A_{t}\right\}_{t \in \mathbb{R}_{+}}$, taking values in a nonempty closed interval $\mathcal{X}$ of $\mathbb{R}$ with (possibly infinite) endpoints $\underline{x}<\bar{x}$, and following the dynamic equation

$$
\begin{aligned}
d X_{t} & =\mu\left(X_{t}, A_{t}\right) d t+\sigma\left(X_{t}, A_{t}\right) d B_{t} \\
X_{0} & =x
\end{aligned}
$$

Assumption 1 There exists a nonempty compact metric space $\mathcal{K}$ such that $A_{t} \in \mathcal{K}$ for all $t .15$

A control process is said to be admissible if it is adapted to the filtration $\left\{\mathcal{F}_{t}\right\}_{t \in \mathbb{R}_{+}}$, satisfies Assumption 1, and Equation (1) has a unique strong solution ${ }^{16}$ The set of admissible control processes is denoted by $\mathcal{A}$.

Given an admissible control $A$, the agent's expected payoff is given by

$$
v(x, A)=E\left[\int_{0}^{\kappa} e^{-r t} f\left(X_{t}^{A}, A_{t}\right) d t+e^{-r \kappa} g\left(X_{\kappa}^{A}\right)\right],
$$

where $X_{t}^{A}$ is the process starting at $x$ and controlled by $A, f\left(X_{t}^{A}, A_{t}\right)$ is the flow payoff at time $t$, $\kappa=\inf \left\{t: X_{t} \notin(\underline{x}, \bar{x})\right\}$ is the first time at which the boundary of $\mathcal{X}$ is hit, and $g(\underline{x})$ and $g(\bar{x})$ are given constants 17 Assumptions 2 and 3 , stated shortly, guarantee that the expected payoff is well defined for any admissible control (see Lemma 1 below).

\footnotetext{
${ }^{14}$ We refer the reader to Karatzas and Shreve (1998) for the standard concepts used in this paper.

${ }^{15}$ The assumption that $\mathcal{K}$ is independent of $x$ is only for expositional simplicity. The analysis can be extended to the case in which the control set depends on $x$, provided that i) for each $x, \mathcal{K}(x)$ is a nonempty, closed subset of the compact metric space $\mathcal{K}$, and ii) the correspondence $x \mapsto K(x)$ is continuous. See Footnote 28 Alternatively, the compactness assumption on $\mathcal{K}$ can be dropped, for example to allow for an unbounded control space, provided that the functions $f, \mu$, and $\sigma$ are bounded in $a$ and uniformly continuous in $(x, a)$. See Appendix $\mathrm{C}$

${ }^{16}$ This definition of admissibility is the one used, among others, by the classic control theory textbook of Fleming and Soner (1993). See also Pham's (2009) recent textbook.

${ }^{17}$ We follow the usual convention of setting $e^{-r \kappa} g\left(X_{\kappa}^{A}\right)=0$ whenever $\kappa=\infty$.
} 
The (optimal) value function ${ }^{18}$ of the problem starting at $x$, denoted $v(x)$, is defined by

$$
v(x)=\sup _{A \in \mathcal{A}} v(x, A) .
$$

An admissible control is said to be optimal if $v(x, A)=v(x)$.

Assumption 2 There exists $K>0$ such that, for all $\left(x, x^{\prime}, a\right) \in \mathcal{X}^{2} \times \mathcal{K}$,

$$
\left|\mu(x, a)-\mu\left(x^{\prime}, a\right)\right|+\left|\sigma(x, a)-\sigma\left(x^{\prime}, a\right)\right|+\left|f(x, a)-f\left(x^{\prime}, a\right)\right| \leq K\left|x-x^{\prime}\right|,
$$

and the functions $\mu(x, \cdot), \sigma(x, \cdot), f(x, \cdot)$ are continuous in a, for each $x{ }^{19}$

The last assumption contains several bounds on the primitives: standard linear growth conditions, a uniform lower bound on $\sigma$, and a condition guaranteeing that, for any control, the state grows at a rate slower than the discount rate.

Assumption 3 There exist constants $K_{1}^{\mu}, K_{2}^{\mu}, K^{\sigma}, K^{f}$, and $\underline{\sigma}$ such that ${ }^{20} K_{2}^{\mu}<r$ and $0<\underline{\sigma} \leq$ $|\sigma(x, a)|,|\mu(x, a)| \leq K_{1}^{\mu}+K_{2}^{\mu}|x|,|\sigma(x, a)| \leq K^{\sigma}(1+|x|)$, and $|f(x, a)| \leq K^{f}(1+|x|)$ for all $(x, a) \in \mathcal{X} \times \mathcal{K}$.

\section{Twice Differentiability of the Value Function}

Our objective is to prove that the value function is twice differentiable in the interior of $\mathcal{X}$ and solves the Hamilton-Jacobi-Bellman (HJB) equation

$$
0=\sup _{a \in \mathcal{K}}-r v(x)+f(x, a)+\mu(x, a) v^{\prime}(x)+\frac{1}{2} \sigma^{2}(x, a) v^{\prime \prime}(x)
$$

with given boundary conditions

$$
v(\underline{x})=g(\underline{x}) \text { if } \underline{x} \text { is finite, and } v(\bar{x})=g(\bar{x}) \text { if } \bar{x} \text { is finite. }
$$

THEOREM 1 Under Assumptions 13 , the following holds:

i) The value function is finite and has linear growth: $|v(x)| \leq K_{v}(1+|x|)$ for some positive constant $K_{v}$.

\footnotetext{
${ }^{18}$ To avoid confusion, we reserve the expression "value function" to the optimal expected payoff, and use the expression "expected payoff" when the control is arbitrary.

${ }^{19}$ When $\mathcal{K}$ is a finite set, the continuity assumption is vacuous.

${ }^{20}$ The condition $K_{2}^{\mu}<r$ can be dropped if $f$ is bounded. In that case, the control problem is equivalent to one in which $X$ is replaced by a smooth increasing transformation $Y$ of $X$, that satisfies this condition, without affecting other conditions.
} 
ii) The HJB equation has a twice continuously differentiable solution which has linear growth.

iii) Any solution to the HJB equation that has linear growth is equal to the value function. In particular, the value function is twice continuously differentiable and is the unique solution with linear growth of the HJB equation.

Before going into the proof of Theorem 1, let us comment briefly on the necessity of our assumptions. First, if $\sigma$ is not bounded away from zero, the value function need not be differentiable, and the HJB equation need not have a classical solution ${ }^{21}$ Moreover, existence and uniqueness of solutions for the controlled SDE (weak or strong) may not be guaranteed. Second, if either $\mu$ or $\sigma$ violate the linear growth condition, the state $X_{t}$ may explode in finite time. Similarly, if the payoff function $f$ violates the linear growth condition, the value function may be infinite even if the state grows at a slower rate than the discount rate ${ }^{22}$ To apply the techniques of Section 4 where we prove the existence of a strong solution, the Lipschitz assumption on $\sigma$ cannot be easily relaxed (see Footnote 42]. The Lipschitz conditions on $f, \mu$, and $\sigma$ are also used to guarantee uniqueness and continuity of solutions to the ODE problems that arise in several proofs of this paper.

Finiteness is only an issue when $\mathcal{X}$ is unbounded, and follows from the following lemma, proved in the Appendix.

Lemma 1 For any admissible control $A$, initial condition $x$, and $\tilde{r}>K_{2}^{\mu}$, we have:

i) $\lim _{t \rightarrow+\infty} E\left|X_{t}^{A}\right| e^{-\tilde{r} t}=0$, and ii) $E\left[\int_{0}^{\infty} e^{-\tilde{r} t}\left|f\left(X_{t}^{A}, A_{t}\right)\right| d t\right]<+\infty$.

This lemma, combined with the linear growth condition on $f$ and the fact that $K_{2}^{\mu}<r$ immediately implies that $v$ is finite and satisfies a linear growth condition 23

The rest of the proof consists of the following steps: 1) Prove the existence of a solution, $w$, to the HJB equation, which has linear growth; 2) Construct a control process based on this solution; 3) Prove that the solution is equal to the value function of the problem and that either the control constructed in 2) is optimal, or that it can be approximated by a sequence of admissible controls.

\footnotetext{
${ }^{21}$ It is, for example, easy to construct such cases when $\sigma$ is identically equal to zero, at least for some control value. Many studies of viscosity solutions deal with dynamic equations for deterministic problems that fail to have a classical solution. If $\sigma$ vanishes only at some point ( $\operatorname{such}$ as $x=0$ ) and otherwise satisfies an ellipticity condition, particular techniques may be used to extend the result. One such example is contained in Appendix D

${ }^{22}$ For example, suppose that $\left\{X_{t}\right\}_{t \geq 0}$ is a geometric Brownian motion with growth rate $\mu<r$ and volatility $\sigma>0$ and that the payoff function is given by $f(x)=x^{2}$ (no control). Then, the value function is infinite if $\mu+\sigma^{2} / 2>r$, as is easily checked.

${ }^{23}$ Precisely, it is shown in Lemma 1 that $E\left|X_{t}^{A}\right|$ is bounded above by $\left(|x|+1+K_{X}\right) e^{K_{2}^{\mu} t}$, uniformly in $A$. This, and the linear growth condition on $f$ guarantee that $f\left(X_{t}, A_{t}\right)$ grows at most at rate $K_{2}^{\mu}<r$, uniformly in $A$. This implies that the integral payoff is bounded linearly in $x$, uniformly in $A$. The terminal payoff is bounded above by $\max \left\{g(\underline{x}) 1_{\underline{x}>-\infty}, g(\bar{x}) 1_{\bar{x}<+\infty}\right\}$, which is also finite.
} 
These steps will imply that any solution of the HJB equation with linear growth must coincide with the value function of the problem and, therefore, will show the uniqueness claimed in Part iii) of Theorem 1 .

We first show the existence of a solution to the HJB equation. This result follows from Proposition 1 below, which is proved in the Appendix. The proposition relies on the following conditions for an arbitrary real-valued function $\bar{H}(x, p, q)$ defined on $\mathcal{X} \times \mathbb{R}^{2}$.

Condition 1 On any compact interval $\mathcal{X}_{0}$ of $\mathcal{X}$, there exist constants $M$ and $K$ such that ${ }^{24}$ for all $(x, p, q) \in \mathcal{X} \times \mathbb{R}^{2}$

i) $|\bar{H}(x, p, q)| \leq M(1+|p|+|q|)$,

ii) $|\bar{H}(x, p, q)-\bar{H}(x, \tilde{p}, \tilde{q})| \leq K(|p-\tilde{p}|+|q-\tilde{q}|)$,

iii) $\bar{H}$ is continuous in $x$ for each $(p, q)$.

Condition 2 For all $(x, q), \bar{H}(x, \cdot, q)$ is nonincreasing in $p$.

Condition 3 For each $\bar{K}>0$, there exist $K_{1}, K_{2}>\bar{K}$ such that for all $x \in \mathcal{X}$, and $\varepsilon \in\{-1,1\}$,

$$
\left.\bar{H}\left(x, K_{1}+K_{2}|x|, \varepsilon K_{2}\right)<0 \text { and } \bar{H}\left(x,-K_{1}-K_{2}|x|\right), \varepsilon K_{2}\right)>0 .
$$

Proposition 1 Suppose that $\bar{H}$ satisfies Conditions 1 3. Then, for any finite $\underline{v}$ and $\bar{v}$, there exists a twice continuously differentiable solution to the equation

$$
w^{\prime \prime}+\bar{H}\left(x, w, w^{\prime}\right)=0
$$

which satisfies $w(\underline{x})=\underline{v}$ if $\underline{x}$ is finite, and $w(\bar{x})=\bar{v}$ if $\bar{x}$ is finite. Moreover, there exists a positive constant $K_{v}$ such that

$$
|w(x)| \leq K_{v}(1+|x|) \text { for all } x \in \mathcal{X}
$$

We apply Proposition 1 to Equation (3) by checking Conditions $1-3$.

Proposition 2 Under Assumptions 13 , the HJB equation (3) has a twice continuously differentiable solution $w$ on $\mathcal{X}$, which has linear growth.

Proof. Equation (3) can be rewritten as

$$
w^{\prime \prime}+H\left(x, w, w^{\prime}\right)=0,
$$

\footnotetext{
${ }^{24}$ Parts ii) and iii) imply that $\bar{H}$ is jointly continuous in $(x, p, q)$. Although we do not use this fact explicitly in the proof, it provides a more direct explanation for why the second derivative of the solution is continuous.
} 
where 25

$$
H(x, p, q)=\max _{a \in \mathcal{K}} \frac{2}{\sigma^{2}(x, a)}(-r p+f(x, a)+\mu(x, a) q) .
$$

We show that $H$ satisfies Conditions 1, 2, and 3, Let

$$
h(a, x, p, q)=\frac{2}{\sigma^{2}(x, a)}(-r p+f(x, a)+\mu(x, a) q),
$$

so that $H(x, p, q)=\max _{a \in \mathcal{K}} h(a, x, p, q)$. Assumption 2 and the strictly positive uniform lower bound on $\sigma$ (Assumption 3) guarantee that $\frac{f(x, a)}{\sigma^{2}(x, a)}, \frac{p}{\sigma^{2}(x, a)}$, and $\frac{\mu(x, a)}{\sigma^{2}(x, a)}$ are Lipschitz in $x$ on compact intervals of $\mathcal{X}$, uniformly in $a$. This guarantees that $h$ is continuous in $a$ and Lipschitz in $x$ on any compact interval $\mathcal{X}_{0}$ of $\mathcal{X}$, uniformly in $a$. Moreover, because $r$ and $\sigma^{2}$ are positive, $h$ is decreasing in $p$ and Condition 2 is satisfied.

To verify Condition 1, we use the following inequality ${ }^{26}$

$$
|H(x, p, q)-H(x, \tilde{p}, \tilde{q})| \leq \max _{a \in \mathcal{K}} \frac{2}{\sigma^{2}(x, a)}|(-r p+f(x, a)+\mu(x, a) q)-(-r \tilde{p}+f(x, a)+\mu(x, a) \tilde{q})| .
$$

This implies that

$$
|H(x, p, q)-H(x, \tilde{p}, \tilde{q})| \leq \frac{2}{\underline{\sigma}^{2}}\left(r|p-\tilde{p}|+\left(K_{1}^{\mu}+K_{2}^{\mu}|x|\right)|q-\tilde{q}|\right),
$$

and proves the Lipschitz condition for any compact interval $\mathcal{X}_{0}$ of $\mathcal{X}$. Similarly, the growth condition follows because $\mu$ and $f$ are bounded on any compact support and $\sigma^{2}$ is bounded below by $\underline{\sigma}^{2}>0$.

Continuity of $H$ in $x$, the last part of Condition 1 , is the key to guarantee that the value function is twice differentiable, even when the optimal control jumps. It is due to Berge's Maximum Theorem. Because the objective $h$ is continuous in $a$ and Lipschitz in $x$, uniformly in $a$, it is easy to show that $h$ is jointly continuous in $(x, a){ }^{27}$ Since also the action set $\mathcal{K}$ is compact, the Maximum Theorem applies, proving that $H$ is continuous in $x 28$

There remains to verify that Condition 3 holds. $H\left(x, K_{1}+K_{2}|x|, \varepsilon K_{2}\right)$ is negative whenever

$$
-r\left(K_{1}+K_{2}|x|\right)+K^{f}(1+|x|)+\left(K_{1}^{\mu}+K_{2}^{\mu}|x|\right) K_{2}<0 .
$$

\footnotetext{
${ }^{25}$ Because all functions are continuous in $a, \mathcal{K}$ is compact, and $\sigma$ is bounded below away from zero, the supremum is achieved as a maximum.

${ }^{26}$ More generally, if $H(\theta)=\max _{a \in \mathcal{K}} h(a, \theta)$, one can prove that $|H(\theta)-H(\tilde{\theta})| \leq \max _{a \in \mathcal{K}}|h(a, \theta)-h(a, \tilde{\theta})|$. For example, suppose that $a, \tilde{a}$ maximize $h$ at $\theta$ and $\tilde{\theta}$, respectively. Then, $H(\theta)-H(\tilde{\theta})=h(a, \theta)-h(\tilde{a}, \tilde{\theta}) \leq h(a, \theta)-$ $h(a, \tilde{\theta}) \leq \max _{a \in \mathcal{K}}|h(a, \theta)-h(a, \tilde{\theta})|$. The other inequality is proved similarly.

${ }^{27}$ This is shown as follows. We fix some values for $p$ and $q$ and omit them from the notation. Suppose that $\left(a_{n}, x_{n}\right)$ converges to $(a, x)$. We have $\left|h(x, a)-h\left(x_{n}, a_{n}\right)\right| \leq\left|h(x, a)-h\left(x, a_{n}\right)\right|+\left|h\left(x, a_{n}\right)-h\left(x_{n}, a_{n}\right)\right|$. The first term converges to zero by continuity of $h$ with respect to $a$, while the second term converges to zero because $h$ is Lipschitz in $x$, uniformly in $a$.

${ }^{28}$ Berge's Theorem also applies if the control domain $\mathcal{K}(x)$ depends on $x$ and satisfies the conditions provided in Footnote 15
} 
Since $K_{2}^{\mu}<r$, this inequality holds for all $x \in \mathbb{R}$ (and hence, on $\mathcal{X}$ ) if and only if

$$
K_{2} \geq \frac{K^{f}}{r-K_{2}^{\mu}} \quad \text { and } \quad K_{1}>\frac{K^{f}+K_{1}^{\mu} K_{2}}{r}
$$

Thus the assumptions in Proposition 1 are satisfied, which shows existence of a solution to the HJB equation for arbitrary boundary conditions at $\underline{x}$ and $\bar{x}$, whenever these points are finite.

\section{What role does optimization play for smoothness?}

As mentioned above, continuity of $H$ in $x$ is used to guarantee that the value function is twice differentiable 29 This continuity comes from an application of Berge's Maximum Theorem to the objective function $h$. If, instead of a maximum selection $x \mapsto \hat{a}(x)$ of $h$, we had chosen an arbitrary selection $x \mapsto \tilde{a}(x)$, the resulting function $\tilde{H}(x, p, q)=h(\tilde{a}(x), x, p, q)$ would generally not be continuous in $x$. This explains why the expected payoff is twice differentiable for the optimal control, whereas it may fail to be so for a strictly suboptimal control: recall that the second derivative is given by $v^{\prime \prime}(x)=-H\left(x, v(x), v^{\prime}(x)\right)$. If $H$ is discontinuous in $x$, the value function cannot be twice continuously differentiable, or even twice differentiable there, since the left and right second derivatives at $x$ will be different.

\section{Proof that the candidate solution $w$ is equal to the value function $v$}

We split up the proof into two inequalities.

Lemma 2 Let $w$ be a solution to the HJB equation (3) that has linear growth, and let $v(x, A)$ be the expected payoff given any admissible control $A$. Then, $w(x) \geq v(x, A)$ for all $x \in \mathcal{X}$.

Proof. The proof follows a standard verification argument, which is included here for completeness. For any fixed admissible control $A$ and finite time $T>0$, Itô's formula implies, for the diffusion $X^{A}$ controlled by $A$ and starting at $x$, that

$$
\begin{aligned}
e^{-r\left(T \wedge \kappa_{Y}\right)} w\left(X_{T \wedge \kappa_{T}}^{A}\right)=w(x)+\int_{0}^{T \wedge \kappa_{T}} e^{-r t}\left(-r w\left(X_{t}^{A}\right)+\right. & \left.\mu\left(X_{t}^{A}, A_{t}\right) w^{\prime}\left(X_{t}^{A}\right)+\frac{1}{2} \sigma^{2}\left(X_{t}^{A}, A_{t}\right) w^{\prime \prime}\left(X_{t}^{A}\right)\right) d t \\
& +\int_{0}^{T \wedge \kappa_{n}} e^{-r t} \sigma\left(X_{t}^{A}, A_{t}\right) w^{\prime}\left(X_{t}^{A}\right) d B_{t}, \quad(8)
\end{aligned}
$$

where $\kappa_{T}$ is the first time that $X_{t}^{A}$ leaves $\mathcal{X} \cap[x-T, x+T]$ and $T \wedge \kappa_{T}=\min \left\{T, \kappa_{T}\right\}$. We have $\kappa=\lim _{T \rightarrow \infty} \kappa_{T}$. The term $e^{-r t} \sigma\left(X_{t}^{A}, A_{t}\right)$ is square integrable over $\left[0, T \wedge \kappa_{T}\right]$ (see Appendix A)

\footnotetext{
${ }^{29}$ This condition is sufficient but not necessary: what matters is that the curve $x \mapsto\left(x, v(x), v^{\prime}(x)\right)$ avoids any discontinuity of $H$.
} 
and $w^{\prime}(x)$ is continuous and therefore bounded on $[x-T, x+T]$. Therefore, the stochastic integral has zero mean. Taking expectations and using (3), we get the inequality

$$
E\left[\int_{0}^{T \wedge \kappa_{T}} e^{-r t} f\left(X_{t}^{A}, A_{t}\right) d t\right] \leq w(x)-E\left[e^{-r\left(T \wedge \kappa_{T}\right)} w\left(X_{T \wedge \kappa_{T}}^{A}\right)\right] .
$$

The linear growth condition satisfied by $w$, along with Lemma 1 , guarantees that $\lim _{T \rightarrow \infty} E\left[e^{-r T} w\left(X_{T}^{A}\right)\right]=$ 0 . Taking the limit of $(9)$ as $T$ goes to infinity ${ }^{30}$ and using the equality $w\left(X_{\kappa}^{A}\right)=g\left(X_{\kappa}^{A}\right)$ yields $v(x, A) \leq w(x)$.

For the reverse inequality, we first obtain a candidate optimal control $A_{t}^{*}$ from the solution to the HJB equation $w$. This candidate need not be admissible, because the stochastic differential equation (1) may fail to have a unique strong solution. We will use a result by Nakao (1972), who has shown ${ }^{31}$ that a one-dimensional SDE has a unique strong solution if its drift is measurable and its volatility has bounded variation and is bounded away from zero 32 We exploit this property to construct an approximation to the candidate control which is admissible and gets arbitrarily close to the desired inequality.

Lemma 3 Let $w$ be a solution to the HJB equation (3) that has linear growth. Then $w(x) \leq v(x)$ for all $x \in \mathcal{X}$.

Proof. We construct a candidate optimal control as follows. Take a solution to the HJB equation $w$, and define $\mathcal{M}(x) \subset \mathcal{K}$ as the set of maximizers of the equation

$$
r w(x)=\max _{a \in \mathcal{K}} f(x, a)+\mu(x, a) w^{\prime}(x)+\frac{1}{2} \sigma(x, a)^{2} w^{\prime \prime}(x) .
$$

The objective is continuous in $a$ and in $x$, and $\mathcal{K}$ is nonempty and compact. The measurable maximum theorem 33 thus guarantees the existence of a measurable selection $\hat{a}(x) \in \mathcal{M}(x)$ of maximizers.

If the control $A_{t}^{*}=\hat{a}\left(X_{t}\right)$ is admissible, applying the previous verification argument, this time with an equality, shows that $w(x)=v\left(x, A^{*}\right)$ and, hence, $w(x) \leq v(x)$. In general, the volatility function $\hat{\sigma}(x)=\sigma(x, \hat{a}(x))$ can jump, violating the standard Lipschitz (or Hölder) continuity conditions usually assumed for the existence of a strong solution and, hence, for admissibility of the control 34 We circumvent this issue by the following approximation argument.

\footnotetext{
${ }^{30}$ The expectation of the integral converges, since the integrand goes to zero at a geometric rate, by Lemma 1

${ }^{31}$ More precisely, Nakao established pathwise uniqueness of weak solutions. This, combined with a major result due to Yamada and Watanabe (1971), shows the existence of a (unique) strong solution. See also Veretennikov (1981) and Revuz and Yor (2001, p. 392) for related results.

${ }^{32}$ Drift and volatility must also be bounded, which holds here for any compact interval of $\mathcal{X}$.

${ }^{33}$ See Aliprantis and Border (1999), p. 570.

${ }^{34}$ See, e.g., Karatzas and Shreve (1998), Chapter 5.2, Theorem 2.5.
} 
Fix any $\varepsilon>0$ and consider a grid of $\mathcal{X}$ with equally spaced intervals of length $\eta$, to be chosen shortly. We define the Markovian control $\tilde{a}$ by $\tilde{a}(x)=\hat{a}(\chi(x))$ where $\chi(x)$ is the element of the grid closest to $x{ }^{35}$ By construction, $\tilde{a}$ is piecewise constant. By Nakao (1972), the SDE

$$
d X_{t}=\mu\left(X_{t}, \tilde{a}\left(X_{t}\right)\right) d t+\sigma\left(X_{t}, \tilde{a}\left(X_{t}\right)\right) d B_{t}
$$

has a unique strong solution, because the function $x \mapsto \sigma(x, \tilde{a}(x))$ has bounded variation. Letting $\left\{\tilde{X}_{t}\right\}_{t \in \mathbb{R}_{+}}$denote this solution, the control $\left\{\tilde{A}_{t}\right\}_{t \in \mathbb{R}_{+}}$defined by $\tilde{A}_{t}=\tilde{a}\left(\tilde{X}_{t}\right)$ is admissible. If $\mathcal{X}$ is compact, the function $f(x, a)+\mu(x, a) w^{\prime}(x)+\frac{1}{2} \sigma(x, a)^{2} w^{\prime \prime}(x)-r w(x)$ is uniformly continuous in $(x, a)$ on $\mathcal{X} \times \mathcal{K}$. Therefore, we can choose a grid mesh $\eta$ small enough to guarantee that

$$
\begin{aligned}
& f(x, \tilde{a}(x))+\mu(x, \tilde{a}(x)) w^{\prime}(x)+\frac{1}{2} \sigma(x, \tilde{a}(x))^{2} w^{\prime \prime}(x)-r w(x) \\
& \geq f(\chi(x), \tilde{a}(x))+\mu(\chi(x), \tilde{a}(x)) w^{\prime}(\chi(x))+\frac{1}{2} \sigma(\chi(x), \tilde{a}(x))^{2} w^{\prime \prime}(\chi(x))-r w(\chi(x))-\varepsilon \geq-\varepsilon .
\end{aligned}
$$

Plugging this inequality in the verification argument based on (8), yields $v(x, \tilde{A}) \geq w(x)-\varepsilon / r$. Since $v(x) \geq v(x, \tilde{A})$, taking the limit as $\varepsilon$ goes to zero yields the desired inequality $v(x) \geq w(x)$.

We now show the result if $\mathcal{X}$ is unbounded, focusing on the case in which $\mathcal{X}=\mathbb{R}$ (the case in which $\mathcal{X}$ is a half-line is treated similarly). To each $\mathcal{X}_{n}=[-n, n]$ corresponds a modulus of continuity $\eta_{n}$ such that 12 holds on $\mathcal{X}_{n}$ if the grid has mesh $\eta_{n}$ and $\tilde{a}$ is constructed as before. We construct $\tilde{a}$ on $\mathcal{X}_{1}$ by using the grid with mesh $\eta_{1}$ on that domain, then extend it on $\mathcal{X}_{2} \backslash \mathcal{X}_{1}$ by using the grid with mesh $\eta_{2}$ on that domain, and so on. This construction defines $\tilde{a}$ over $\mathbb{R}$. Moreover, $\tilde{a}$ is piecewise constant with finitely many jumps over any $\mathcal{X}_{n}$, and hence has bounded variation over any compact interval of $\mathbb{R}$. Finally, 12 holds, by construction, on the entire domain $\mathbb{R}$. The rest of the argument is unchanged.

\subsection{Example: Optimal Growth}

The analysis of growth models typically relies on some smoothness of the value function, as the success of the Benveniste-Scheinkman Theorem (1979) illustrates. When time is continuous, smoothness takes on a particularly important role: it guarantees that the Bellman equation is satisfied everywhere, and, hence, that it can be directly used to derive properties of optimal policies ${ }^{36}$ In models without uncertainty, concavity assumptions are often required to establish differentiability (see Benveniste-Scheinkman (1979) and, more recently, Rincon-Zapatero and Santos (2009, 2010)).

\footnotetext{
${ }^{35}$ We can adopt any arbitrary convention when there are two points of the grid that are closest to $x$.

${ }^{36}$ One illustration is provided by Quah and Strulovici (2013, who show monotonicity of the capital growth in the discount factor. Their analysis connects supermodularity properties of the objective function in the HJB equation.
} 
Theorem 1 shows that such assumptions can be dispensed with when uncertainty is modeled by Brownian noise.

Consider an agent with initial capital $x$ who seeks to maximize his lifetime utility by managing his capital through consumption, savings, and effort. Formally, the agent solves

$$
\sup _{A \in \mathcal{A}} E\left[\int_{0}^{\infty} e^{-r s} u\left(X_{s}^{A}, A_{s}\right) d s\right]
$$

subject to

$$
d X_{t}^{A}=\mu\left(X_{t}^{A}, A_{t}\right) d t+\sigma\left(X_{t}^{A}, A_{t}\right) d B_{t},
$$

where the control domain $\mathcal{K}$ is a compact subset of $\mathbb{R}^{k}$ for some $k \geq 1$, and the drift and volatility functions $\mu$ and $\sigma$ are such that $X$ is always nonnegative and that 0 is an absorption point, with $u(0, a)=\mu(0, a)=\sigma(0, a)=0$ for all $a$. The dimensions of $A$ can represent consumption, leisure and effort, technological choices, etc. $X_{t}^{A}$ is the capital available to the agent at time $t$.

We do not place any other restriction on the agent's technology and utility functions apart from the Lipschitz and linear growth conditions required by Theorem 1. By assuming that, for each $\varepsilon>0, \sigma(x, a)$ is uniformly bounded away from zero on $[\varepsilon,+\infty) \times \mathcal{K}$, we can apply ${ }^{37}$ Theorem 1 to show that the value function is $C^{2}$ on $(\varepsilon,+\infty)$ for each $\varepsilon>0$ and, hence, on the interior $(0,+\infty)$ of the entire domain. Moreover, it solves for all $x>0$ the HJB equation

$$
r v(x)=\max _{a \in \mathcal{K}} u(x, a)+\mu(x, a) v^{\prime}(x)+\frac{1}{2} \sigma^{2}(x, a) v^{\prime \prime}(x) .
$$

In Appendix D, we allow the state space $\mathcal{X}$ to stretch all the way to 0 , and the volatility function $\sigma$ to vanish at zero, and show how our arguments extend to that case.

A major issue in such environment is to establish the existence of an optimal strategy for the agent. Section 4 provides general results which may be used in this setting. If the control $A$ consists only of a consumption choice, then the volatility $\sigma$ is typically independent of $A$ : the agent's consumption affects the dynamics of the capital $X$ only by reducing its drift. In that case, Corollary 1 of Section 4 implies that any measurable maximizing selection $a(x)$ of 13 defines an admissible, optimal control. Section 4.1 analyzes existence for a more general control process.

\subsection{Example: Multi-Armed Bandit}

Consider a multi-armed bandit problem with a common unknown parameter $\tilde{\theta} \in\left\{\theta_{L}, \theta_{H}\right\}$, representing a binary, payoff relevant state of the economy. The agent must choose at each time an

\footnotetext{
${ }^{37}$ The termination value $g(\varepsilon)$ in $[2]$ is set to the actual value function $v(\varepsilon)$, so that the value function on $[\varepsilon,+\infty)$ coincides, on that domain, with the value function of the initial control problem over the entire domain $[0,+\infty)$.
} 
arm $i$ in some compact set $\mathcal{K}$, given his belief $X_{t} \in[0,1]=\operatorname{Pr}\left(\tilde{\theta}=\theta_{H} \mid \mathcal{F}_{t}\right)$ about $\tilde{\theta}$. Given the choice $A_{t}$ of an arm at time $t$, the agent learns about $\tilde{\theta}$ according to some equation 38

$$
d X_{t}^{A}=\mu\left(X_{t}^{A}, A_{t}\right) d t+\sigma\left(X_{t}^{A}, A_{t}\right) d B_{t}
$$

Because the belief is a martingale, $\mu$ is identically equal to zero. Moreover, from standard computations (see, e.g., Bolton and Harris (1999)), the volatility has the form $\sigma\left(X_{t}, A_{t}\right)=X_{t}\left(1-X_{t}\right) \xi\left(A_{t}\right)$ and is thus entirely characterized by the vector $\{\xi(i)\}_{i \in \mathcal{K}} . \xi(i)$ is the signal-to-noise ratio of the aggregate signal generated by $\operatorname{arm} i$. The expected flow payoff from playing arm $i$ at time $t$ if the current belief is $x$ is

$$
f\left(X_{t}^{A}, A_{t}\right)=E\left[\pi(\tilde{\theta}, i) \mid X_{t}^{A}=x, A_{t}=i\right]
$$

where $\pi$ is the expected flow payoff (or utility) if the state of the economy is $\tilde{\theta}$ and the agent has pulled arm $i$. This flow is necessarily linear in $x: f(x, i)=x \pi\left(\theta_{H}, i\right)+(1-x) \pi\left(\theta_{L}, i\right)$.

The assumptions of Theorem 1 are satisfied over any domain $\mathcal{X}_{\varepsilon}=(\varepsilon, 1-\varepsilon)$ with $\varepsilon \in(0,1 / 2)$. Therefore, Theorem 1 implies that $v$ is twice differentiable and solves the HJB equation over any such domain and, therefore, over $(0,1)$.

This shows that the value function of any Brownian multi-armed bandit problem in which the payoff distributions of all arms depend on the same unknown parameter is twice continuously differentiable and solves the HJB equation. This result implies, as shown in Appendix E, that with finitely many arms, the number of switching points between arms is finite (and bounded above by a function that depends only on the number of arms), and that the optimal control is well defined. It is also used to construct an example with four arms in which a given arm is used on disjoint subsets of the belief domain.

With infinitely many arms, or if the agent can allocate divisible resources across arms, it is a priori unclear whether there exists a well defined optimal control to this problem. Section 4.2 provides a positive answer for a resource allocation problem with two arms.

\section{Existence of an Optimal Control}

The control constructed in the proof of Lemma 3 need not be admissible because it may fail to generate a strong solution to the SDE (1) ${ }^{39}$ In the context of Examples 3.1 and 3.2, the trajectories

\footnotetext{
${ }^{38}$ Unlike a setting in which arms are independent, such as the one analyzed by Gittins (1979), here all arms relate to the same state. Analyzing control problems with multidimensional states is beyond the scope of this paper.

${ }^{39}$ Standard results, e.g. Karatzas and Shreve (1998), Th. 5.15, guarantee that the constructed control generates a weak solution of SDE (1). As argued earlier, we think that strong solutions are a more natural concept in most
} 
of the optimal capital stock or the experimenter's belief may have no solution or have a weak solution which cannot be pinned down given the history of the Brownian Motion. This section provides conditions for the existence of a maximizing selection that yields an admissible optimal control. All the results of this section are based on the following theorem, which provides a novel and straightforward application of Nakao (1972)'s main result.

THEOREM 2 Suppose that the selection $\hat{a}$ of maximizers is such that the function $\hat{\sigma}: x \mapsto \sigma(x, \hat{a}(x))$ has locally bounded variation. Then, the control $A_{t}^{*}$ that it generates (as defined by Lemma 3), is admissible and optimal.

Proof. Admissibility follows from Nakao (1972), who has shown that a one-dimensional SDE has a unique strong solution if its drift is measurable and its volatility has bounded variation and is bounded away from zero (see Footnote 31 for more details on this result). Optimality follows from a standard verification argument.

The bounded variation condition is necessary for the result: Barlow (1982) provides a class of stochastic differential equations for which the volatility does not have bounded variation and there does not exist a strong solution, even if the volatility is bounded below, away from zero 40

An easy case in which $\hat{\sigma}$ has bounded variation is if $\sigma(x, a)$ is independent of $a$, a case that arises in many economic applications, such as the consumption choice problem of Section 3.1 .

Corollary 1 Suppose that $(x, a) \mapsto \sigma(x, a)$ is independent of a. Then, the control $A_{t}^{*}$ generated by the selection $\hat{a}$ of maximizers (as given by Lemma 3) is admissible and optimal.

Proof. The function $x \mapsto \sigma(x)$ is Lipschitz in $x$, by Assumption 3, and has therefore bounded variation. The result then follows from Theorem 2 ,

Beyond Corollary 1, the volatility $\sigma$ may depend on a subset, $\alpha$, of the control. In that case, one way of guaranteeing that $\hat{\sigma}$ has bounded variation is to check that the correspondence $\mathcal{M}$ of maximizers has a selection $\hat{a}=(\hat{\alpha}, \hat{\beta}) \in \mathcal{K}_{1} \times \mathcal{K}_{2} \subset \mathbb{R}^{k_{1}} \times \mathbb{R}^{k_{2}}$, such that $\hat{\alpha}$ has bounded variation 41

economic applications and provide a natural continuous-time counterpart of discrete time models. With a strong solution, there exists a measurable map $\Phi:\left(C\left(\mathbb{R}_{+}, \mathbb{R}\right), \mathcal{B}\right) \rightarrow\left(C\left(\mathbb{R}_{+}, \mathbb{R}\right), \mathcal{B}\right)$, where $\mathcal{B}$ is the Borel algebra on $\mathbb{R}$, such that the state trajectory is given by $X(\omega)=\Phi(B(w))$ for a.e. $\omega$ (see, e.g., Cherny (2002)). Such map does not exist for weak-only solutions: knowledge of the Brownian path and of the agent's strategy does not suffice to determine the state trajectory. Instead, the filtration must be enlarged to account for unmodeled uncertainty, in a way that is endogenous and may depend on the parameters of the problem.

${ }^{40}$ We are grateful to Nicolai Krylov for pointing this reference out to us.

${ }^{41}$ See Ambrosio and Dal Maso (1990) for the definition of bounded variation for functions defined and taking values in Euclidean spaces. Continuity of the control is neither necessary nor sufficient, as it does not imply bounded variation. 
and to assume that $\sigma(x, \alpha)$ is uniformly Lipschitz continuous over $\mathcal{X}_{0} \times \mathcal{K}_{1}$ for any compact interval $\mathcal{X}_{0}$ of $\mathcal{X}$. This implies that $\hat{\sigma}$ has bounded variation, as guaranteed by the following result (see Ambrosio and Dal Maso (1990)).

Proposition 3 Suppose that $\sigma$ is uniformly Lipschit $t^{42}$ continuous on $\mathcal{X}_{0} \times \mathcal{K}_{1}$ for any compact interval $\mathcal{X}_{0}$ of $\mathcal{X}$, and that there exists a selection $\hat{a}(\cdot)=(\hat{\alpha}(\cdot), \hat{\beta}(\cdot))$ of $\mathcal{M}(\cdot)$ such that $\hat{\alpha}$ has bounded variation. Then, $\hat{\sigma}$ has bounded variation.

Guaranteeing that the correspondence $\mathcal{M}$ has a selection with bounded variation would a priori seem a benign and easily satisfied requirement. However, we are not aware of any general result of this kind 43 Similarly, it seems difficult in general to show the existence of an absolutely continuous selection.

Fortunately, there are more specific strategies to guarantee that the control has bounded variation. If the state space $\mathcal{X}$ can be broken down into finitely many intervals over each of which there is a selection of maximizers such that each component of $\alpha$ is either monotonic or differentiable, this will guarantee the existence of a selection with bounded variation over the entire domain. 44

THEOREm 3 Suppose that $\sigma$ is uniformly Lipschitz continuous on $\mathcal{X}_{0} \times \mathcal{K}_{1}$, for any compact interval $\mathcal{X}_{0}$ of $\mathcal{X}$, and that $\mathcal{X}$ can be decomposed into disjoint intervals $\left\{\mathcal{X}_{j}\right\}_{j=1}^{n}$ over each of which there is a selection $\hat{a}^{j}=\left(\hat{\alpha}^{j}, \hat{\beta}^{j}\right)$ of $\mathcal{M}$ such that each component of $\hat{\alpha}^{j}$ is either monotonic or differentiable with locally bounded derivative ${ }^{45}$ Then, there exists an optimal control, which is Markov and characterized by the selector $\hat{a}$ defined by $\hat{a}(x)=\hat{a}^{j}(x)$ for $x \in \mathcal{X}_{j}$.

Proof. Fixing $j$, let $\left\{\chi_{i}\right\}_{i=0}^{m}$ denote a partition of some compact interval $\mathcal{I}$ of $\mathcal{X}_{j}$ and $\left\{\hat{\alpha}_{k}^{j}\right\}_{k=1}^{k_{1}}$ denote the first $k_{1}$ components of $\hat{a}^{j}$. We have

$$
\sum_{i=0}^{m-1}\left|\hat{\sigma}\left(\chi_{i+1}\right)-\hat{\sigma}\left(\chi_{i}\right)\right| \leq K \sum_{i=0}^{m-1}\left(\left|\chi_{i+1}-\chi_{i}\right|+\sum_{k=1}^{k_{1}}\left|\hat{\alpha}_{k}^{j}\left(\chi_{i+1}\right)-\hat{\alpha}_{k}^{j}\left(\chi_{i}\right)\right|\right)
$$

\footnotetext{
${ }^{42}$ The assumption that $\sigma$ is Lipschitz cannot be easily relaxed: Josephy (1981, Theorem 4) has shown that for the composition $f \circ g$ to have bounded variations for all functions $g$ with bounded variation, $f$ must be Lipschitz.

${ }^{43}$ Chistyakov (2004) defines a concept of bounded variation for correspondences, for which he proves selection theorems guaranteeing a selection of bounded variation. However, we are not aware of any work connecting Chistyakov's concept with the correspondence of maximizers in optimization problems. We are grateful to Vyacheslav Chistyakov for his insights into this problem.

${ }^{44}$ Another, weaker requirement, is to show the existence of a selection that is $K$-lower or $K$-upper Lipschitz. This property implies that the selection can only jump in a single direction and implies bounded variation. It can be established by applying the monotone comparative statics techniques described in this section to a suitable change of variable. See Amir and De Castro (2013) and the references therein.

${ }^{45}$ This condition means that the derivative is bounded on any compact interval, and holds if the relevant components of $\hat{\alpha}^{j}$ are continuously differentiable.
} 
where $K$ is the Lipschitz constant of $\sigma$ over $\mathcal{I} \times \mathcal{K}_{1}$. Each $\hat{\alpha}_{k}^{j}$ is monotonic or differentiable with locally bounded derivative and, hence, has bounded variation. This and (14) show that $\hat{\sigma}$ has bounded variation over each $\mathcal{X}_{j}$ and, hence, over $\mathcal{X}$. The result then follows from Theorem 2 .

Sections 4.1 and 4.3 provide applications of Theorem 3 in which $\alpha$ is differentiable. Unfortunately, differentiability is violated in many settings, most obviously when the control set $\mathcal{K}$ is discrete. Monotonicity offers an alternative way of exploiting Theorem 3. In many economic problems, the optimal control is monotonic in the state: consumption is increasing in wealth, investment decreases with risk, etc. Establishing such monotonicity without knowing the objective function explicitly is precisely the focus of the theory of monotone comparative statics, as described below.

In what follows, we focus on the case in which $\mathcal{K}_{1}$ is an interval of $\mathbb{R}$ (i.e., $k_{1}=1$ ). A function $\rho(x, a)$ is supermodular on some domain $\mathcal{X}_{0} \times \mathcal{K}_{1}$ if for all $\tilde{a} \geq a$ in $\mathcal{K}_{1}$ and $\tilde{x} \geq x$ in $\mathcal{X}_{0}, \rho(x, a)+\rho(\tilde{x}, \tilde{a}) \geq$ $\rho(\tilde{x}, a)+\rho(x, \tilde{a})$, and submodular if the reverse inequality holds on that domain (or, equivalently, if $-\rho$ is supermodular). When $\rho$ is differentiable in $a$, supermodularity is equivalent to $\rho_{a}$ being nondecreasing in $x$. When $\rho$ is twice differentiable, supermodularity is equivalent to the cross partial being everywhere nonnegative. Supermodularity is a sufficient condition for the maximizer correspondence $x \mapsto \mathcal{M}(x)$ to be nondecreasing in the strong set order, which means that for all $x \leq \tilde{x}, a \in \mathcal{M}(x)$, and $\tilde{a} \in \mathcal{M}(\tilde{x})$, we have $\min \{a, \tilde{a}\} \in \mathcal{M}(x)$ and $\max \{a, \tilde{a}\} \in \mathcal{M}(\tilde{x})$. In particular, the selections constructed from the smallest and largest maximizers, respectively, are nondecreasing for any supermodular function, and nonincreasing for any submodular one 46

The following result focuses for simplicity on the case in which $\alpha=a$, so that $\mathcal{K}_{1}=\mathcal{K} \subset \mathbb{R}$.

COROLlary 2 Suppose that $\sigma$ is uniformly Lipschitz continuous on $\mathcal{X}_{0} \times \mathcal{K}$, for any compact interval $\mathcal{X}_{0}$ of $\mathcal{X}$, and let

$$
\rho(x, a)=\frac{1}{\sigma^{2}(x, a)}\left(-r v(x)+f(x, a)+\mu(x, a) v^{\prime}(x)\right),
$$

where $v$ is the value function of the problem, and suppose that $\mathcal{X}$ can be decomposed into $n$ consecutive intervals $\left\{\mathcal{X}_{j}\right\}_{j=1, \cdots, n}$ such that on each product $\mathcal{X}_{j} \times \mathcal{K}, \rho$ is either supermodular or submodular. Then, there exists an optimal control, which is Markov and characterized by a selector $\hat{a}$ of $\mathcal{M}$ such that $\hat{a}$ has bounded variation.

Proof. From (6), maximizing $\rho$ with respect to $a$ is equivalent to solving the maximization problem in the HJB equation. Let $\hat{a}(x)=\max \mathcal{M}(x)$. Whether $\rho$ is supermodular or submodular on $\mathcal{X}_{j} \times \mathcal{K}$, $\hat{a}$ is monotonic on this interval. The result follows from Theorem 3 ,

\footnotetext{
${ }^{46}$ This result is easy to check. See Topkis (1978) or Milgrom and Shannon (1994) for a proof. The strong set order is also called the Veinott set order (see Veinott, 1989).
} 
REMARK 1 For any strictly positive function $\zeta(x, a)$, note that the HJB equation

$$
0=\max _{a \in \mathcal{K}}-r v(x)+f(x, a)+\mu(x, a) v^{\prime}(x)+\frac{1}{2} \sigma^{2}(x, a) v^{\prime \prime}(x)
$$

has exactly the same maximizers as the equation

$$
0=\max _{a \in \mathcal{K}} \zeta(x, a)\left(-r v(x)+f(x, a)+\mu(x, a) v^{\prime}(x)+\frac{1}{2} \sigma^{2}(x, a) v^{\prime \prime}(x)\right) .
$$

Such transformations are useful for proving monotonicity or differentiability of a selection of maximizers 47

In many economic problems (such as experimentation problems, or when the agent has an increasing concave utility flow), it is possible to show that the value function $v$ has a constant sign, is monotonic, and is either convex or concave 48 This yields the following application of Corollary 2 . Let $l(x, a)=1 / \sigma^{2}(x, a), \bar{f}=l f$, and $\bar{\mu}=l \mu$. Those functions are all primitives of the control problem.

COROllary 3 Suppose that i) $\sigma$ is uniformly Lipschitz continuous on $\mathcal{X}_{0} \times \mathcal{K}$, for any compact interval $\mathcal{X}_{0}$ of $\mathcal{X}$, ii) $v$ is nonnegative, nondecreasing, and convex, and iii) $\bar{\mu}$ is nondecreasing in a and supermodular, $\bar{f}$ is supermodular, $l$ nonincreasing in a and submodular. Then, there exists an optimal control, and this control is Markov and nondecreasing in $x$.

The corollary is straightforward to prove: its conditions guarantee that each term in 15 is supermodular.

The supermodularity assumed in Corollary 2 can be weakened in several ways. Indeed, it suffices that $\rho$ satisfies the single-crossing property or Interval Dominance Order (IDO) property in $(a, x)$ for asserting the existence of a monotonic selection on any given interval 49 Here is a useful way of checking these properties: when $\rho$ is differentiable with respect to $a$, the IDO property is guaranteed to hold $\sqrt{50}$ over $\mathcal{K} \times \mathcal{I}$, where $\mathcal{I}$ is any interval of $\mathcal{X}$, if there exists a positive, nondecreasing function

\footnotetext{
${ }^{47}$ The transformation $\zeta(x, a)=2 / \sigma^{2}(x, a)$ removes $v^{\prime \prime}(x)$ from the maximization problem, and was used for (6) and Corollary 2 Another example, when $\mu$ is known to be strictly positive, is to use $\zeta(x, a)=1 / \mu(x, a)$. This permits to take $v^{\prime}$ out of the maximization problem.

${ }^{48}$ There are many techniques to establish this, either by analyzing the HJB equation, or by constructing various controls to directly show that the value function must be increasing and convex. Applications in this paper provide several such examples.

${ }^{49}$ The single-crossing property (Milgrom and Shannon, 1994), generalizes supermodularity (Topkis, 1978), and is itself generalized by the IDO property (Quah and Strulovici, 2009). Comparative statics also obtain for objective functions that are quasi-concave and ordered by the location of their peaks, as studied by Karlin and Rubin (1956) and Lehmann (1988). This latter ordering is also generalized by the IDO property.

${ }^{50}$ See Quah and Strulovici (2009, Proposition 2).
} 
$\gamma_{\mathcal{I}}(\cdot)$ of $a$ such that, for each $x^{\prime \prime}>x^{\prime}, \rho_{a}\left(x^{\prime \prime}, a\right) \geq \gamma_{\mathcal{I}}(a) \rho_{a}\left(x^{\prime}, a\right)$. If $\gamma_{\mathcal{I}}$ is constant over $\mathcal{K}$, the inequality implies that $\rho$ satisfies the single crossing property in $(a, x)$. If $\gamma_{\mathcal{I}}$ is identically equal to 1 , we recover the supermodularity condition.

\subsection{Example: Optimal Growth with Consumption and Portfolio Optimization}

We reconsider the example of Section 3.1. The agent chooses, in addition to consumption, the fraction $\varphi_{t} \in[0,1]$ of his wealth going to a risky asset, so that $A_{t}=\left(C_{t}, \varphi_{t}\right){ }^{51}$ The volatility $\sigma\left(X_{t}, A_{t}\right)$ is now increasing in $\varphi_{t}$, and we cannot apply Corollary 1 anymore to prove the existence of an optimal control. However, if one can show that the agent's risk-taking behavior is nondecreasing in his capital holdings, this will imply monotonicity of $\varphi$, and guarantee the existence of an optimal control, by Theorem 3 . To guarantee nonnegativity of the capital process $X$, we assume that the agent cannot consume when $x=0$. This does not affect Theorem 1, as long as the feasible consumption set $[0, \bar{c}(x)]$ is continuous in $x$

Slightly simplifying the setting, the HJB equation for this problem is

$$
r v(x)=\max _{(c, \varphi) \in[0, \bar{c}(x)] \times[0,1]}\left\{u(c)+[\mu(x, \varphi)-c] v^{\prime}(x)+\frac{1}{2} \sigma^{2}(x, \varphi) v^{\prime \prime}(x)\right\} .
$$

Thus, the optimal $\varphi$ maximizes the objective

$$
\mu(x, \varphi) v^{\prime}(x)+\frac{1}{2} \sigma^{2}(x, \varphi) v^{\prime \prime}(x) .
$$

If this objective function satisfies the single crossing property in $(\varphi, x)$, there must exist a nondecreasing maximizing selection $\varphi$. If $\sigma$ is Lipschitz continuous in $\varphi$, Theorem 3 then implies that there exists an optimal control.

The single crossing property holds if i) $v$ is increasing and concave ${ }^{53}$ ii) $\mu$ and $\sigma$ are increasing in $\varphi$, and iii) for any $\varphi_{1}<\varphi_{2}$

$$
\frac{-v^{\prime \prime}(x) \Delta \sigma^{2}(x)}{v^{\prime}(x) \Delta \mu(x)}
$$

is decreasing in $x$, where $\Delta \sigma^{2}(x)=\sigma^{2}\left(x, \varphi_{2}\right)-\sigma^{2}\left(x, \varphi_{1}\right)$ and $\Delta \mu(x)=\mu\left(x, \varphi_{2}\right)-\mu\left(x, \varphi_{1}\right)$. Single crossing is then a direct consequence of Proposition 1 in Quah and Strulovici (2012) 54 In turn, (16)

\footnotetext{
${ }^{51}$ We are ruling out short sales and borrowing, which guarantees that $\varphi_{t}$ lies in the compact space $[0,1]$.

${ }^{52}$ See Footnotes 15 and 28 . We can, for example, take $\bar{c}(x)$ to be any continuous function that rapidly increases from $\bar{c}(0)=0$ to some positive constant $\bar{c}$. For the concavity argument below (Footnote 57), we also require that $\bar{c}(\cdot)$ be concave.

${ }^{53}$ Such properties may be shown from direct arguments, as in Footnote 57.

${ }^{54}$ Conditions i) and ii) guarantee that the functions $v^{\prime \prime}(x) \Delta \sigma^{2}(x)$ and $v^{\prime}(x) \Delta \mu(x)$ each have a constant sign and, thus, are single crossing functions. Condition iii) is the signed-ratio monotonicity condition in Quah and Strulovici (2012), which guarantees that the sum is also a single crossing function.
} 
holds if $-v^{\prime \prime}(x) / v^{\prime}(x)$ and $\Delta \sigma^{2}(x) / \Delta \mu(x)$ are both decreasing. The first condition means that the agent has decreasing absolute risk aversion in $x$, while the second condition is immediate to check for any given drift and volatility functions $\mu$ and $\sigma 55$ Thus, we recover the intuition, stated above, that if the agent's risk-aversion is decreasing in his capital, the control $\varphi$ is monotonic and there exists an optimal control.

The previous result relied on the value function exhibiting decreasing absolute risk aversion, which is an endogenous property. The next approach avoids this problem. It proves differentiability of $\varphi$, exploiting another possibility offered by Theorem 3 .

We specialize the model to a version of Merton's consumption and investment problem 56 The safe asset has a constant return $\mu_{0}$, while the a risky asset has a payoff that follows a geometric Brownian motion with a higher return $\mu>\mu_{0}$. We impose the condition $\mu<r$, which is standard in the portfolio optimization literature and is equivalent to the inequality $K_{2}^{\mu}<r$ of Assumption 3 . The agent's capital follows the equation

$$
d X_{t}=\left[X_{t}\left(\left(\mu-\mu_{0}\right) \varphi_{t}+\mu_{0}\right)-C_{t}\right] d t+X_{t} \varphi_{t} \sigma d B_{t}
$$

The agent has a twice differentiable flow utility $u(\cdot)$ such that $u^{\prime}(c)>0$ and $u^{\prime \prime}(c)<0$ for all $c \geq 0$. The HJB equation is

$$
r v(x)=\max _{(c, \varphi) \in[0, \bar{c}(x)] \times[0,1]} u(c)+\left(x\left(\mu-\mu_{0}\right) \varphi+x \mu_{0}-c\right) v^{\prime}(x)+\frac{1}{2} x^{2} \sigma^{2} \varphi^{2} v^{\prime \prime}(x) .
$$

It is easily shown that $v^{\prime}(x)>0$. The optimal consumption $c(x)$ therefore satisfies the first-order condition

$$
u^{\prime}(c)=v^{\prime}(x)
$$

or equals the corner value $\bar{c}(x)$. It is also easily shown that the value function is concave ${ }^{57}$ There is a unique maximizer $\varphi(x)$ to the HJB equation, given by 58

$$
\varphi(x)=\min \left\{-\frac{v^{\prime}(x)}{x v^{\prime \prime}(x)} \frac{\mu-\mu_{0}}{\sigma^{2}}, 1\right\} .
$$

\footnotetext{
${ }^{55}$ That condition can be interpreted as a sort of increasing marginal Sharpe ratio: the additional exposure to risk from moving from $\varphi_{1}$ to $\varphi_{2}$, has a better Sharpe ratio for higher capital levels than lower ones.

${ }^{56}$ See Merton $(1969,1971)$ and Duffie $(2001)$ for a general presentation.

${ }^{57}$ Concavity is established as follows: for $0<x^{1}<x^{2}$ and $\lambda \in(0,1)$, let $x=\lambda x^{1}+(1-\lambda) x^{2}$. Consider any admissible controls $\varphi^{1}, C^{1}$ and $\varphi^{2}, C^{2}$ chosen starting from $x^{1}$ and $x^{2}$, respectively. Let $\varphi_{t}=\left(\lambda X_{t}^{1} \varphi_{t}^{1}+(1-\right.$ $\left.\lambda) X_{t}^{2} \varphi_{t}^{2}\right) /\left(\lambda X_{t}^{1}+(1-\lambda) X_{t}^{2}\right) \in[0,1]$ and $C_{t}=\lambda C_{t}^{1}+(1-\lambda) C_{t}^{2}$. It is easy to check that the admissible control $(\varphi, C)$ starting from $x$ yields $X_{t}=\lambda X_{t}^{1}+(1-\lambda) X_{t}^{2} \geq 0$ for all $t$. Moreover, $u\left(C_{t}\right) \geq \lambda u\left(C_{t}^{1}\right)+(1-\lambda) u\left(C_{t}^{2}\right)$, by concavity of $u$. Discounting and integrating proves the claim.

${ }^{58}$ If $v^{\prime \prime}(x)=0$, the first term of the minimization is assumed to be equal to $+\infty$.
} 
Proposition 4 The maximizing selection $(c(x), \varphi(x))$ of the HJB equation generates an admissible, optimal control.

Proof. It suffices to show that $\varphi$ has bounded variation on $\mathcal{I}_{\varepsilon}=\{x \in(\varepsilon, 1 / \varepsilon): \varphi(x) \in(0,1)\}$, which consists of disjoint open intervals, for each $\varepsilon<1$. Following Theorem 3 , it suffices to show that $\varphi$ is continuously differentiable on that domain. Rewriting the HJB equation, we hav£ 59

$$
v^{\prime \prime}(x)=-\max _{(c, \varphi) \in[0, \bar{c}(x)] \times[0,1]}\left\{\frac{2}{x^{2} \sigma^{2} \varphi^{2}}\left(u(c)+\left(x\left(\mu-\mu_{0}\right) \varphi+x \mu_{0}-c\right) v^{\prime}(x)-r v(x)\right)\right\} .
$$

Since the maximizers $c(x)$ and $\varphi(x)$ are unique, Corollary 4, Part iii) of Milgrom and Segal (2002) implies that $v^{\prime \prime}$ is differentiable at all $x>0$. Moreover, the derivative is continuous, as is easily checked. Therefore, (18) implies that $\varphi$ is continuously differentiable on $\mathcal{I}_{\varepsilon}$.

\subsection{Example: Multi-Armed Bandit and Resource Allocation}

We now reconsider the bandit problem of Section 3.2 with two arms, but in which the agent has a fixed resource, normalized to 1 , to allocate between the arms at each time 60 As before, each arm has a payoff distribution that depends on the common parameter $\tilde{\theta}$ and the state at time $t$ is the agent's belief $X_{t}=\operatorname{Pr}\left(\tilde{\theta}=\theta_{H} \mid \mathcal{F}_{t}\right)$. The control of the agent is now a fraction $A_{t} \in[0,1]$ allocated to the first arm. The total payoff from both arms in each state is $\pi\left(\theta, A_{t}\right)$. Notice that each allocation $a \in[0,1]$ yields a combination of two signals which may be aggregated into a single signal with volatility $\sigma(x, a)=\xi(a) x(1-x)$ about the parameter $\tilde{\theta}$, and yields an expected payoff

$$
f(x, a)=x \pi\left(\theta_{H}, a\right)+(1-x) \pi\left(\theta_{L}, a\right) .
$$

Thus, we are exactly in the setting of Section 3.2 , with $\mathcal{K}=[0,1]$. In particular, the value function of the problem is twice continuously differentiable and solves everywhere on $(0,1)$ the HJB equation

$$
0=\max _{a \in[0,1]} f(x, a)+\frac{1}{2}(x(1-x))^{2} \xi^{2}(a) v^{\prime \prime}(x)-r v(x) .
$$

This equation may be rewritten as

$$
v^{\prime \prime}(x)=\frac{2}{(x(1-x))^{2}} \max _{a \in[0,1]}\left\{\frac{1}{\xi^{2}(a)}(f(x, a)-r v(x))\right\} .
$$

Therefore, the existence of a monotone optimal selection will be guaranteed if the function

$$
\frac{-r v(x)}{\xi^{2}(a)}+\frac{f(x, a)}{\xi^{2}(a)}
$$

\footnotetext{
${ }^{59}$ The optimal allocation $\varphi(x)$ is strictly positive for all $x$, since $v^{\prime}(x)>0$.

${ }^{60}$ Examples of experimentation with resource allocations include Bergemann and Välimäki (1997, 2000), Bolton and Harris (1999), and Keller et al. (2005). In the last setting, one arm is safe, whereas both arms are "risky" in the present model.
} 
is either supermodular or submodular in $(a, x)$.

Assuming that $\Delta f(a)=f(1, a)-f(0, a) \geq 0$ for all $a$ (i.e., $\theta_{H}$ is the "good" state of the economy), $v$ is nondecreasing in $x$. Therefore, the first term in 22 is supermodular (submodular) if and only if $\xi(a)$ is increasing (decreasing) in $a$. Similarly, the second term is supermodular (submodular) in $(x, a)$ if $\Delta f(a) / \xi^{2}(a)$ is increasing (decreasing) in $a$. Combining these observations with Theorem 3 proves the following result.

Proposition 5 Suppose that $\xi(a)$ and $\Delta f(a) / \xi^{2}(a)$ are either both increasing or both decreasing in a. Then there exists a monotone optimal selection $a(x)$ and, therefore, an optimal control.

\subsection{Existence of an Optimal Contract in Principal Agent Problems}

This application revisits the analysis contained in Sannikov (2008). The objective here is to provide conditions under which the optimal contract characterized in that paper generates a strong solution for the continuation value process of the agent. The existence problem discussed here is actually relevant for many recent continuous-time principal-agent models. In the recursive approach to principal-agent models, the principal is viewed as "controlling" the continuation value of the agent by providing him with consumption, and rewarding or punishing him depending on his output. If the continuation value process admits only a weak solution, the principal may implicitly be randomizing in the contract, as the continuation value is not necessarily determined by the history of output alone. This raises several issues regarding the interpretation of the continuation value process as well as the principal's risk aversion, as we argue below.

To save space, we only sketch the presentation and arguments already contained in Sannikov (2008), and refer the reader to that paper for a detailed exposition.

The principal chooses a consumption process $\left\{C_{t}\right\}_{t \geq 0}$ and can implement, by choosing the right incentives, some effort process $\left\{A_{t}\right\}_{t \geq 0}$. Precisely, assuming that the agent's continuation utility $W_{t}$ is adapted to the filtration generated by the exogenous uncertainty, the Martingale Representation Theorem implies that

$$
d W_{t}=\left(r W_{t}-u\left(C_{t}\right)+h\left(A_{t}\right)\right) d t+\psi\left(A_{t}\right) d B_{t}
$$

where $A_{t}$ is the level of effort that the principal chooses to implement, $u$ and $h$ are the utility and effort cost functions of the agent, and $\psi(a)=h^{\prime}(a)$ is the contract sensitivity to observed output.

The principal seeks to maximize the payoff

$$
F_{0}=E\left[\int_{0}^{\tau} e^{-r t}\left(A_{t}-C_{t}\right) d t\right]
$$


subject to (23) and some initial participation constraint $W_{0}=w 6$

Therefore, the principal faces an optimal control problem in which the state is $W_{t}$ and the controls are $C_{t}$ and $A_{t}$, following the standard notation in principal-agent models ${ }^{62}$ Sannikov has shown that the agent is "retired" when $W_{t}$ hits some upper bound $\bar{w}$ and fired when $W_{t}$ hits zero. The optimal consumption level $c(w)$ is easily shown to be bounded by some level $\bar{c}$ on $[0, \bar{w}]$, while the effort domain is assumed to be bounded above by some level $\bar{a}$.

The principal's HJB equation on $(0, \bar{w})$ is

$$
r F(w)=\max _{(a, c) \in[0, \bar{a}] \times[0, \bar{c}]}\left\{a-c+F^{\prime}(w)(r w-u(c)+h(a))+\frac{1}{2} \psi(a)^{2} F^{\prime \prime}(w)\right\} .
$$

Under standard assumptions on $u$ and $h$, Sannikov shows that the HJB equation has a solution $F$ that is twice continuously differentiable. Moreover, $F$ is strictly concave.

At the optimal contract, standard results imply that there exists a weak solution to the SDE (23), whenever $\psi(a(w))$ is bounded away from zero 63 With a weak solution, the continuation value process is not necessarily adapted to the filtration generated by the Brownian Motion $B_{t}$, which implies that there is more randomness in $W_{t}$, and therefore the contract, than generated by the output process.

One possible interpretation for having (only) a weak solution is that the principal is randomizing over continuation utility levels. However, the principal's objective $F(w)$ is strictly concave, which implies that he is risk-averse along the optimal contract. This makes this interpretation problematic as a justification for a weak solution. The uncertainty arising from the broader filtration implied by weak solutions seems conceptually different from the one obtained if the principal explicitly allows all contractual variables to depend on other sources of uncertainty, such as a second Brownian motion.

Another issue is that, as observed by Sannikov (2008, Footnote 29), the application of the Martingale Representation Theorem, which is a key step in the analysis, must also be modified to include the larger filtration. In that case, the resulting representation of $W$ cannot a priori be reduced to a stochastic integral with respect to the initial Brownian motion. As a result, the analysis of the contract, which was largely based on choosing the integrand of that stochastic integral, must be modified. Intuitively, it raises the issue of what "sensitivity" the principal should apply to the new sources of uncertainty. Guaranteeing a strong solution gets rid of these issues.

\footnotetext{
${ }^{61}$ The stopping time $\tau$ is time the agent is either retired or fired.

${ }^{62}$ Thus, what has been called " $x$ " in earlier section is now " $w$ ", whereas " $A$ " now refers to only one component of the bidimensional control. $F$, instead of $v$ denotes the value function of the problem.

${ }^{63}$ See, e.g., Revuz and Yor (2001, Corollary 1.12, p. 372).
} 
Proposition 6, below, provides simple conditions on the effort cost function $h(a)$ under which the continuation value process has a unique strong solution. Thus, $W_{t}$ is adapted to $\mathcal{F}_{t}^{B}$, and the contract does not involve randomization.

Proposition 6 Suppose that the effort cost function $h$ is increasing, three times differentiable, and satisfies $h(0)=0, h^{\prime}(0)>0, h^{\prime \prime}(a)>0$ and $h^{\prime \prime \prime}(a) \geq 0$ for all $a \in(0, \bar{a}]$. Then, the SDE

$$
d W_{t}=\left(r W_{t}-u\left(C_{t}\right)+h\left(A_{t}\right)\right) d t+\psi\left(A_{t}\right) d B_{t}
$$

has a unique strong solution.

The assumptions on $h$ are satisfied, for example, if $h(a)=\gamma_{1} a^{p}+\gamma_{0} a$ for $p \geq 2$ and $\gamma_{0}, \gamma_{1}>0$, as in Sannikov's (2008) numerical example, if it is exponential, or is equal to any positive combination of such functions.

Proof. Because consumption appears only in the drift of (23), it suffices to show that $a(w)$ has bounded variation. The optimality equation for $a$ can be reduced to the optimization problem

$$
\max _{a \in[0, \bar{a}]} a+F^{\prime}(w) h(a)+F^{\prime \prime}(w) \frac{1}{2} \psi(a)^{2} .
$$

We will first show that the objective function in 25) is strictly quasiconcave in $a$, for each $w$. That is, its derivative with respect to $a, \Psi(a, w)=1+F^{\prime}(w) h^{\prime}(a)+F^{\prime \prime}(w) \psi^{\prime}(a) \psi(a)$, can cross 0 at most once as $a$ increases, from above. We have, recalling that $\psi(a)=h^{\prime}(a)$,

$$
\Psi_{a}(a, w)=F^{\prime}(w) h^{\prime \prime}(a)+F^{\prime \prime}(w)\left(h^{\prime \prime \prime}(a) h^{\prime}(a)+h^{\prime \prime}(a)^{2}\right)
$$

Our assumptions imply that $h^{\prime}(a)>0$ for all $a$. We can rewrite the previous equation as

$$
\Psi_{a}(a, w)=\frac{\Psi(a, w)-1}{h^{\prime}(a)} h^{\prime \prime}(a)+F^{\prime \prime}(w) h^{\prime \prime \prime}(a) h^{\prime}(a) .
$$

Whenever $\Psi(a, w) \leq 0$, the previous equation implies that

$$
\Psi_{a}(a, w) \leq-\frac{h^{\prime \prime}(a)}{h^{\prime}(a)}+F^{\prime \prime}(w) h^{\prime \prime \prime}(a) h^{\prime}(a) .
$$

Since $h^{\prime \prime}(a)>0$ and $h^{\prime \prime \prime}(a) \geq 0$ for $a>0$, and $F$ is concave, we conclude that $\Psi_{a}(a, w)<0$ whenever $\Psi(a, w) \leq 0$ and $a>0$, proving strict quasiconcavity of the objective function in (25).

This also shows uniqueness of the optimizer $a(w)$. Moreover, $a(w)$ is continuous in $w$, from (25) and Berge's maximum theorem, whose conditions are easily checked here. Therefore, it suffices to show that $a(w)$ has bounded variation on any interval $\left(w_{1}, w_{2}\right)$ over which $a(w) \in(0, \bar{a})$. By Theorem 3, we will have proved the result if we show that $a(w)$ is continuously differentiable on any such interval. 
The optimal effort is determined on $\left(w_{1}, w_{2}\right)$ by the first-order condition $\Psi(a(w), w)=0$. To show continuous differentiability of $a(w)$, we first observe that the optimal consumption $c(w)$ is also unique, as an immediate consequence of (24) and the fact that $u^{\prime}(c)$ is strictly decreasing in $c$. An envelope theorem of Milgrom and Segal (2002, Corollary 4, Part iii)) then implies that $F$ is three times continuously differentiable and, hence, that $\Psi$ is differentiable with respect to $w \longdiv { 6 4 }$ Because $\Psi_{a}(a, w)$ is strictly negative, evaluated at $a(w)$, the implicit function theorem can be applied to the first-order condition, showing that $a$ is continuously differentiable in $w$ on the desired domain.

\section{Optimal Stopping and Smooth Pasting}

Optimal stopping problems appear in many economic settings such as labor search, experimentation with bandits, the exercise of real options, and the optimal choice of capital structure with endogenous bankruptcy. The smooth pasting property, which states that the value function must be continuously differentiable everywhere, can yield conditions which uniquely determine the optimal stopping region. Typically, researchers apply guess and verify, that is, they assume that the smooth pasting condition holds, use it to find the optimal stopping region, and verify that this solution equals the optimal value function 65

This section establishes, under conditions similar to those of Section 2 , that the value function of any optimal stopping problem is continuously differentiable and provides a verification result. In particular, the value function is differentiable at any threshold at which stopping becomes optimal, which is the smooth pasting property 66 For clarity, we separate optimal control and optimal stopping problems. The problems can be combined with an appropriate extension of Theorems 1 and $4^{67}$ As will be clear from the arguments below, there is a strong relation between the proofs

\footnotetext{
${ }^{64}$ The envelope theorem is applied to the modified HJB equation $F^{\prime \prime}(w)=-\max _{a, c}\left\{2 / \psi(a)^{2}\left[a-c+F^{\prime}(w)(r w-\right.\right.$ $u(c)+h(a))-r F(w)]\}$. Our assumptions on $h$ guarantee that $\psi(a)=h^{\prime}(a)$ is bounded below by $h^{\prime}(0)>0$ and, hence, that the previous equation holds on $\left(w_{1}, w_{2}\right)$.

${ }^{65}$ This is done for example by Leland (1994) in the context of optimal bankruptcy, Bolton and Harris (1999) and Moscarini and Smith (2001) for experimentation problems, McDonald and Siegel (1986) for real options, and Jovanovic (1979) for labor search.

${ }^{66}$ Given our continuity and linear growth assumptions on $f(x)$ and $g(x)$, we can show that the optimal stopping time equals the first hitting time of the set $\{x \in \mathcal{X}: v(x)=g(x)\}$. See Peskir and Shiryaev (2006), Corollary 2.9, p.46.

${ }^{67}$ In the combined problem, the optimal continuation region consists of disjoint open intervals, as in this section, and on any such interval, the analysis of Section 2 can be applied, showing that value function of the controlled process is twice differentiable. The analysis of the present section can then be replicated to show that the value function is everywhere $C^{1}$, replacing the dynamic equation 27 by the HJB equation, which is satisfied on the optimal continuation region.
} 
of Theorems 1 and 4

Consider the optimal stopping problem

$$
v(x)=\sup _{\tau \in \mathcal{T}} E\left[\int_{0}^{\tau} e^{-r t} f\left(X_{t}\right) d t+e^{-r \tau} g\left(X_{\tau}\right)\right],
$$

where $\mathcal{T}$ is the set of all stopping times adapted to the initial filtration $\mathcal{F}$ and valued in $[0, \infty]$, and $\left\{X_{t}\right\}_{t \in \mathbb{R}_{+}}$solves the equation

$$
d X_{t}=\mu\left(X_{t}\right) d t+\sigma\left(X_{t}\right) d B_{t}
$$

subject to the initial condition $X_{0}=x$. We maintain the same assumptions as before on $\mu, \sigma$ and $f$, which guarantee that the SDE has a unique strong solution, and, along with Assumption 4, that the expected payoff is well defined for all stopping times.

Assumption $4 g$ is $C^{1}$ and $|g(x)| \leq K^{g}(1+|x|)$ for some constant $K^{g} \geq 0$.

TheOREM 4 Under Assumptions 2- 4, the following holds. 69

i) The value function is finite and has linear growth: $|v(x)| \leq K_{v}(1+|x|)$ for some positive constant $K_{v}$.

ii) $v$ is continuously differentiable on the interior of $\mathcal{X}$ and satisfies $v(x) \geq g(x)$ for all $x \in \mathcal{X}$.

iii) Let $\mathcal{Y}$ denote the subset of $\mathcal{X}$ for which $v(x)=g(x)$. Then $\mathcal{Y}$ is closed and $v$ solves the following equation on $\mathcal{X} \backslash \mathcal{Y}$ :

$$
w^{\prime \prime}(x)+\frac{1}{\frac{1}{2} \sigma(x)^{2}}\left(-r w(x)+f(x)+\mu(x) w^{\prime}(x)\right)=0 .
$$

Proof. The proof of i) is similar to i) of Theorem 1. The subset of $\mathcal{Y}$ consists of all the states at which it is optimal to stop immediately. By continuity ${ }^{70}$ of $v$ and $g, \mathcal{X} \backslash \mathcal{Y}$ consists of disjoint open intervals $\left\{\mathcal{Z}_{i}\right\}_{i \in I}$. Pick any two points $x_{1}<x_{2}$ in such an interval. The result stated in Appendix B guarantees the existence of a $C^{2}$ solution $w$ to the ordinary differential equation

$$
w^{\prime \prime}(x)+\frac{1}{\frac{1}{2} \sigma(x)^{2}}\left(-r w(x)+f(x)+\mu(x) w^{\prime}(x)\right)=0
$$

\footnotetext{
${ }^{68}$ For example, like Theorem 1 the proof below exploits existence and uniqueness of solutions to particular BVP, as well as their continuity with respect to initial conditions.

${ }^{69}$ The pure stopping problem that we consider is equivalent to reducing the control set $\mathcal{K}$ of Section 2 to a singleton.

${ }^{70}$ Continuity of $v$ can be shown by first principles. It follows from the Feller continuity of the diffusion process and continuity of terminal payoff function. An elementary argument in the absence of flow payoffs can be found in Peskir and Shiryaev (2006, p. 154).
} 
with boundary conditions $w\left(x_{1}\right)=v\left(x_{1}\right)$ and $w\left(x_{2}\right)=v\left(x_{2}\right)$. A standard verification argument then shows that $v$ coincides with $w$ on any such interval and, therefore, that $v$ is $C^{2}$ on such interval and, hence, on $\mathcal{X} \backslash \mathcal{Y}=\cup_{i \in I} \mathcal{Z}_{i}$, showing iii).

The key is to show that ii) holds at any boundary point of $\mathcal{Y}$, which is the smooth-pasting property. Consider now the boundary of some interval $\mathcal{Z}_{i}$, for example the upper boundary, and call it $x^{*}$. By construction, $v(x)>g(x)$ for $x$ in a left-neighborhood of $x^{*}$, and $v\left(x^{*}\right)=g\left(x^{*}\right)$. In particular, $v_{l}^{\prime}\left(x^{*}\right)$, the left derivative of $v$ at $x^{*}$, must be less than or equal to $g^{\prime}\left(x^{*}\right)$.

To show that the inequality is tight, suppose, by contradiction, that $v_{l}^{\prime}\left(x^{*}\right)<g^{\prime}\left(x^{*}\right)$, and consider the domain $\left[x_{1}, x_{2}^{*}=x^{*}+\varepsilon\right]$ for some $x_{1}$ in $\mathcal{Z}_{i}$ and some small $\varepsilon>0$. From Appendix B, there exists a solution $w$ to Equation (27) on $\left[x_{1}, x_{2}^{*}\right]$, with initial value $w\left(x_{1}\right)=v\left(x_{1}\right)$ and initial slope $w^{\prime}\left(x_{1}\right)=v^{\prime}\left(x_{1}\right)$. Moreover, this solution satisfies $w\left(x^{*}\right)=v\left(x^{*}\right)$, and $w^{\prime}\left(x^{*}\right)=v_{l}^{\prime}\left(x^{*}\right)$, because $v$ solves the same initial value problem (IVP) on the domain $\left[x_{1}, x^{*}\right]$, and the solution is unique (see Lemma 4). Therefore, $w(x)<g(x)$ for $x$ in a right neighborhood of $x^{*}$, and without loss, on $\left(x^{*}, x_{2}^{*}\right)$. Taking a slightly higher slope $\hat{s}>s^{*}$, consider the solution $\hat{w}$ to the IVP on the domain $\left[x_{1}, x_{2}^{*}\right]$ with initial slope $\hat{s}$ and initial value $v\left(x_{1}\right)$. For $\hat{s}$ close to $s$, this solution hits $g$ at some $\hat{x} \in\left(x_{1}, x_{2}^{*}\right)$, because solutions to the IVP are continuous in $s$ (see Lemma 5 in the Appendix). Moreover Lemma 11, also proved in the Appendix, implies that $\hat{w}(x)>w(x)$ for all $x \in\left(x_{1}, x^{*}\right]$ and, therefore, that $\hat{x}>x^{*}$. We redefine $\hat{w}$ by setting $\hat{w}(x)=g(x)$ for all $x>\hat{x}$. By construction, $\hat{w}\left(x^{*}\right)>g\left(x^{*}\right)=v\left(x^{*}\right)$. Moreover, $\hat{w}$ corresponds to the expected payoff obtained if the following stopping strategy is used: starting from $x^{*}$, continue until either $x_{1}$ or $\hat{x}$ is reached. If $\hat{x}$ is reached first, stop. If $x_{1}$ is reached first, follow the initial strategy leading to value $v\left(x_{1}\right)$. This strategy thus gives, starting from $x^{*}$, a strictly higher expected payoff than $v\left(x^{*}\right)$, yielding a contradiction.

This shows that $v$ is differentiable at $x$ in the following cases: i) $x$ lies in the interior of some interval $\mathcal{Z}_{i}$, ii) $x$ connects two intervals $\mathcal{Z}_{i}$ and $\mathcal{Z}_{j}$ (i.e., it is the upper bound of one interval, and the lower bound of the other), and iii) $x$ is a bound of some interval $\mathcal{Z}_{i}$, and $v(y)=g(y)$ in some neighborhood on the other side of $x$. Moreover, in cases ii) and iii) the derivative is given by $v^{\prime}(x)=g^{\prime}(x)$. In all these cases, the derivative is continuous at $x$ because the solution to $(27)$ on any $\mathcal{Z}_{i}$ is twice differentiable and because $g$ is $C^{1}$.

There remains to show the result when $x$ is an accumulation point of stopping and continuation regions. This is proved in Section F.1 of the Appendix.

The next result is a standard verification argument, which directly follows from a simple adaptation of Lemmas 2 and 3 . The proof is significantly simpler because there is no control, and the hitting time $\kappa$ is now defined as the first time that $X_{t}$ hits $\mathcal{Y}$. We state it here for completeness ${ }^{71}$

\footnotetext{
${ }^{71}$ A proof can also be found in Krylov (1980), p. 41f.
} 
Proposition 7 Suppose that in addition to Assumptions 2-4. $g$ is twice continuously differentiable except on a set of zero measure and $w: \mathcal{X} \rightarrow \mathbb{R}$ has the following properties:

1. $w$ is continuously differentiable, and twice continuously differentiable except on a set of zero measure 72

2. $w(x) \geq g(x)$ for all $x \in \mathcal{X}$.

3. For all $x \in \mathcal{X}$ such that $w$ is twice differentiable,

$$
r w(x) \geq f(x)+\mu(x) w^{\prime}(x)+\frac{1}{2} \sigma^{2}(x) w^{\prime \prime}(x),
$$

with a tight inequality whenever $w(x)>g(x)$

Then, $w$ is the value function of the optimal stopping problem (26).

The assumption that $g$ is smooth even on the optimal stopping region cannot be easily dispensed with. Décamps et al. (2006) provide an example in which the value function of a candidate solution is twice differentiable on the continuation region and satisfies smooth pasting, but the solution is suboptimal since $g$ has a point of non-differentiability on the stopping region.73

\subsection{Example: Option Value}

A simple application of the smooth pasting property is to prove, under great generality, the wellknown principle that when the value function of any stopping problem is convex, the agent waits beyond the myopic optimum to stop, due to an "option value of waiting." This principle arises in investment decisions (Dixit, 1989)), experimentation problems ${ }^{74}$ the exercise of American and real options, and bankruptcy decisions (Leland, 1994).

Formally, suppose that the terminal value function is a constant: $g(x)=\bar{g}$ for all $x$, and suppose that it can be established by some means, that the value function $v$ of the agent is convex. ${ }^{75}$ Consider

\footnotetext{
${ }^{72}$ Those properties are enough to apply Itô's lemma.

${ }^{73}$ Precisely, Décamps et al. correct and extend a result by Dixit (1993b) in which the optimal stopping payoff is the maximum of the values of executing two projects, which is non-differentiable at the point of indifference. We are grateful to an anonymous referee for pointing us to this reference.

${ }^{74}$ In experimentation problems with a risky and a safe arm, moving to the safe arm amounts to a stopping problem. See e.g., Bolton and Harris (1999).

${ }^{75}$ Typical arguments include direct reasoning on the strategy of the agents, or reasoning based on the dynamic equation for $v$. One version of the former approach is similar to the argument given in Footnote 57 , but reversing it: pick any stopping policy that is optimal for $x$, and show that the convex combination of the value starting from $x_{1}<x_{2}$, following $x$, dominates $v(x)$. This will work if the process $X_{t}$ is linear in the initial condition $x$ (as is the case for geometric Brownian motion), and if the flow and terminal payoffs are convex in their argument.
} 
any maximal interval $\mathcal{Z}$ over which continuing is strictly optimal, as in the proof of Theorem 4 , with endpoints $\underline{x}$ and $\bar{x}{ }^{76}$ Theorem 4 implies that for any finite endpoint of $\mathcal{Z}$, say $\bar{x}$, the smooth pasting condition holds, i.e., $v^{\prime}(\bar{x})=g^{\prime}(\bar{x})=0$. Moreover, the Bellman equation on $\mathcal{Z}$ implies that

$$
0=-r v(\bar{x})+f(\bar{x})+\mu(x) v^{\prime}(\bar{x})+\frac{1}{2} \sigma^{2}(x) v_{l}^{\prime \prime}(\bar{x}),
$$

where $v_{l}^{\prime \prime}(\bar{x})$ is the left second derivative of $v$ at $\bar{x}$, which was shown earlier to always exist under the assumptions of Theorem 4. Convexity of $v$ and smooth pasting then imply that

$$
f(\bar{x}) \leq r \bar{g},
$$

with a strict inequality if $v_{l}^{\prime \prime}(\bar{x})>0$. This shows that the option value of waiting is positive. To see this, suppose first that $\bar{g}=0$ (stopping yields a zero lump sum). From (29), the agent stops when his current flow payoff is negative. If $\bar{g}$ is nonzero, the same idea applies, where $r \bar{g}$ is the flow-payoff equivalent of stopping. The result holds for any shape of the stopping region.

\section{Conclusion}

This paper provides unifying and self-contained arguments showing, under simple conditions, the smoothness of value functions and the existence of optimal strategies. These arguments can be extended to analyze situations in which the volatility vanishes over some subset of the state space, the control domain depends on the state, or control and stopping problems are combined. They can also be used to derive specific results in applications, such as bounding the number of switching points in finite multi-armed bandit problems, proving the uniqueness of an optimal threshold in optimal stopping problems, establishing effort monotonicity in a principal-agent model, or differentiability of the optimal strategy in growth or investment models.

The analysis has emphasized the role played by comparative statics for dynamic optimization problems. Envelope theorems were helpful in applications to prove the differentiability of policy functions and the existence of an optimal control. This suggests that these tools, whose use is currently largely circumscribed to economic analysis, have a role to play in control theory, granting them a new, indirect role, for economics.

The assumption of a one-dimensional state space, while accounting for many economic models, is clearly restrictive. For example, we do not consider the case of a state that includes a time dimension or, in multi-armed bandit problems, the case in which each arm has a separate, independent state. Such extensions are special, as only one state evolves stochastically at any given time, which may

\footnotetext{
${ }^{76}$ As shown in that proof, the strict continuation domain always consists of disjoint open intervals.
} 
be helpful in obtaining useful generalizations of the methods. More generally, however, there is a real need in economics for a better understanding of the properties of optimal policies and value functions with a multidimensional state space, both for qualitative analysis and for constructing explicit solutions.

\section{Acknowledgments}

We are grateful for comments from John Quah and Yuliy Sannikov. Part of this research was accomplished while the first author was visiting the Economic Theory Center at Princeton University, whose hospitality is gratefully acknowledged. This author is also thankful for financial support from the NSF under Grant No. 1151410.

\section{A Proof of Lemma 1}

Consider the function $\varphi$ defined on $\mathbb{R}$ by $\varphi(x)=|x|$ for $|x| \geq 1$ and $\varphi(x)=\frac{1}{2}\left(1+x^{2}\right)$ for $|x|<1$. As is easily checked, $\varphi$ is $C^{1}$ everywhere, $C^{2}$ except at -1 and 1 , and satisfies $\left|\varphi^{\prime}(x)\right| \leq 1$ and $|x| \leq \varphi(x) \leq|x|+1$ for all $x \in \mathbb{R}$ and $\left|\varphi^{\prime \prime}(x)\right| \leq 1$ for $x<1$ and $\varphi^{\prime \prime}(x)=0$ for $|x|>1$. By Itô's lemma 77 we have for any admissible control $A$, and $t \geq 0$

$$
\varphi\left(X_{t}^{A}\right)=\varphi(x)+\int_{0}^{t}\left(\mu\left(X_{s}^{A}, A_{s}\right) \varphi^{\prime}\left(X_{s}^{A}\right)+\frac{1}{2} \sigma^{2}\left(X_{s}^{A}, A_{s}\right) \varphi^{\prime \prime}\left(X_{s}^{A}\right)\right) d s+\int_{0}^{t} \sigma\left(X_{s}^{A}, A_{s}\right) \varphi^{\prime}\left(X_{s}^{A}\right) d B_{s} .
$$

Because $|\sigma(x, a)| \leq K^{\sigma}(1+|x|)$, the integrand of the stochastic integral is square integrable over $[0, t]{ }^{78}$ Therefore, the stochastic integral has zero mean. Taking expectations, and using the bounds on $\mu, \sigma$ and $\varphi$ we get, letting $\Phi_{t}=E \varphi\left(X_{t}^{A}\right)$,

$$
\Phi_{t} \leq \Phi_{0}(x)+\int_{0}^{t}\left(\left(K_{1}^{\mu}+K_{2}^{\mu} \Phi_{s}\right)+\frac{1}{2} \tilde{K}\right) d s
$$

where $\tilde{K}=\max _{(x, a) \in[-1,1]] \times \mathcal{K}} \sigma^{2}(x, a)$. By Gröwnwall's lemma, this implies that

$$
\Phi_{t} \leq\left(\Phi_{0}(x)+K_{X} t\right) \exp \left(K_{2}^{\mu} t\right)
$$

where $K_{X}=K_{1}^{\mu}+\tilde{K} / 2$. Since $\left|X_{t}^{A}\right| \leq \varphi\left(X_{t}^{A}\right)$ for all $t$ and $A$, we conclude that $E\left|X_{t}^{A}\right| \leq(|x|+1+$ $\left.K_{X} t\right) \exp \left(K_{2}^{\mu} t\right)$ for all $t$. In particular, $E\left|X_{t}^{A}\right| e^{-r t}$ goes to zero as $t$ goes to infinity. The last claim of the lemma is straightforward to prove, using the linear growth condition on $f$.

\footnotetext{
${ }^{77}$ Itô's lemma applies to any function that is $C^{1}$ and a.e. $C^{2}$.

${ }^{78}$ Precisely, square integrability follows from the inequality $\sigma^{2}\left(X_{s}^{A}, A_{s}\right) \leq 2\left(K^{\sigma}\right)^{2}\left(1+x^{2}\right)$, as well as a standard estimate on $E\left|X_{t}^{A}\right|^{2}$. See Krylov (1980), Corollary 6, p. 81.
} 


\section{B Proof of Proposition 1}

\section{B.1 General Results on Initial Value Problems}

We start with two results pertaining to the existence of solutions to initial value problems (IVP) and their continuity with respect to the initial conditions. We start with some function $\tilde{H}:(x, y) \mapsto \tilde{H}(x, y)$ defined on $\mathcal{X} \times \mathbb{R}^{n}$ and taking values in $\mathbb{R}^{n}$, which satisfies the following condition:

Condition 4 On any compact interval $\mathcal{I}$ of $\mathcal{X}$,

i) $|\tilde{H}(x, y)| \leq M(1+|y|)$,

ii) $\left|\tilde{H}(x, y)-\tilde{H}\left(x, y^{\prime}\right)\right| \leq K\left|y-y^{\prime}\right|$,

iii) $\tilde{H}$ is continuous in $x$ for each $y$.

Lemma 4 If Condition 4 holds, the ordinary differential equation

$$
y^{\prime}(x)=\tilde{H}(x, y(x))
$$

with initial condition $y\left(x_{0}\right)=y_{0}$ has a unique continuously differentiable solution on $\mathcal{X}$, for any $x_{0} \in \mathcal{X}$ and $y_{0} \in \mathbb{R}^{n}$.

Let $y\left(x, y_{0}\right)$ denote the solution to 30 on $\mathcal{X}$ with initial condition $y\left(x_{0}\right)=y_{0}$.

Lemma 5 Given Condition \&, $y\left(\cdot, y_{0}\right)$ is uniformly continuous in $y_{0}$.

The proofs are standard and omitted. For Lemma 4 see Hartman (2002, Theorem 1.1, p. 8) 79 For Lemma 5 see Hartman (2002, Theorem 2.1, p. 94).

\section{B.2 Proof of Proposition 1: Bounded Case}

We specialize the results of Section B.1 to our setting: suppose that $y=(p, q)$ and $H(x, p, q)$ satisfies Conditions 13 In this case, the function $\tilde{H}(x,(p, q))=(q, H(x, p, q))$ satisfies Condition 4 .

The proof of Proposition 1 is based on the "shooting method" (see, e.g., Bailey, 1962). The general intuition for the argument is as follows. We start from some initial conditions $(\underline{x}, \underline{v})$ and consider the solution $w$ to the IVP

$$
w^{\prime \prime}+H\left(x, w, w^{\prime}\right)=0
$$

subject to the initial conditions $w(\underline{x})=\underline{v}$ and $w^{\prime}(\underline{x})=s$. Given our assumptions on $H$, Lemma 4 guarantees that this IVP will have a unique, twice continuously differentiable solution. Lemma 5 guarantees that the solution continuously depends on the starting slope $s$. We can establish the existence of a solution to the

\footnotetext{
${ }^{79}$ That theorem establishes local existence. The growth condition, i), guarantees that the solution can be extended to the entire domain $\mathcal{I}$.
} 
boundary value problem (BVP) if we can show that it is always possible to pick the slope $s$ in such a way that at $\bar{x}$, the solution to the IVP will hit $\bar{v}$.

The proof relies on constructing a particular compact, convex subset of $(x, v)$-plane, ending with a vertical segment at $x=\bar{x}$ that contains $\bar{v}$. We then define a mapping between the initial slope $s$ of the solution to the IVP with initial value $w(\underline{x})=\underline{v}$ and initial slope $s$, and the "last" point at which it hits the boundary, and show that the mapping is onto. That property then proves the existence of an initial slope such that the solution hits the value $\bar{v}$ at $\bar{x}$.

Lemma 6 There exist positive constants $K_{1}, K_{2}$ such that the functions $b_{1}(x)=-K_{1}-K_{2}|x|$ and $b_{2}(x)=$ $K_{1}+K_{2}|x|$ satisfy the inequalities

$$
\begin{aligned}
& b_{1}^{\prime \prime}+H\left(x, b_{1}, b_{1}^{\prime}\right)>0 \\
& b_{2}^{\prime \prime}+H\left(x, b_{2}, b_{2}^{\prime}\right)<0
\end{aligned}
$$

for all $x \neq 0$, and the boundary constraints $\underline{v} \in\left(b_{1}(\underline{x}), b_{2}(\underline{x})\right)$ and $\bar{v} \in\left(b_{1}(\bar{x}), b_{2}(\bar{x})\right)$.

Proof. We have for $x \neq 0$ and $K_{1}, K_{2}$ satisfying Condition 3 ,

$$
\left.b_{2}^{\prime \prime}(x)+H\left(x, b_{2}(x), b_{2}^{\prime}(x)\right)=H\left(x, K_{1}+K_{2}|x|\right), K_{2} \operatorname{sgn}(x)\right)
$$

which is strictly negative. The argument for $b_{1}$ is analogous. The boundary constraints are clearly satisfied provided that $K_{1}$ and $K_{2}$ are large enough, and Condition 3 guarantees that we can choose them that way.

Lemma 7 There exist $s_{1}$ and $s_{2}$ such that the solution to IVP (31) hits $b_{2}$ for all initial slopes $s \geq s_{2}$ and $b_{1}$ for all initial slopes $s \leq s_{1}$.

Proof. By suitably translating the problem, we can without loss assume that $\underline{x}=\underline{v}=0.80$ We wish to show that for high enough initial slopes $s$, the solution $w_{s}$ hits $b_{2}$. Consider the auxiliary IVP

$$
u^{\prime \prime}+K u^{\prime}+H(x, u(x), 0)+\varepsilon=0
$$

subject to $u(0)=0$ and $u^{\prime}(0)=s$, where $K$ is the Lipschitz constant of $H$ and $\varepsilon$ is a positive constant. We will show that, for $s$ high enough, $u$ is strictly increasing on $[0, \bar{x}]$, with a derivative that is bounded below by a linear function of $s$. For fixed $s$, let $\tilde{x}>0$ denote the first time that $u^{\prime}(x)=0$. On $[0, \tilde{x}]$, we have $u(x) \geq 0$. By Condition 2, we have $H(x, u(x), 0) \leq H(x, 0,0)$ on that domain, and

$$
u^{\prime \prime}(x)+K u^{\prime}(x)+M \geq 0,
$$

where $M=\max _{x \in[0, \bar{x}]}|H(x, 0,0)|+\varepsilon>0$. Applying Grönwall's inequality to the function $g(x)=-u^{\prime}(x)-$ $M / K$, which satisfies the inequality $g^{\prime}(x) \leq-K g(x)$ on $[0, \tilde{x}]$, we conclude that

$$
u^{\prime}(x) \geq[s+M / K] \exp (-K x)-M / K
$$

\footnotetext{
${ }^{80}$ The translation is obtained by letting $\bar{w}(x)=w(x-\underline{x})-\underline{v}$ and $\bar{H}\left(x, w, w^{\prime}\right)=H\left(x-\underline{x}, w+\underline{v}, w^{\prime}\right) . \bar{H}$ inherits the Lipschitz and monotonicity properties of $H$, as is easily checked.
} 
on that domain. This implies that $\tilde{x}$ is bounded below by

$$
\frac{1}{K} \log \left(\frac{s+M / K}{M / K}\right)
$$

which exceeds $\bar{x}$ for $s$ high enough. Moreover, the lower bound on $u^{\prime}$ also implies that $u$ hits $b_{2}$ for $s$ large enough and does not cross it again before $\bar{x}$ is reached.

To conclude the proof, we will show that the IVP solution $w$ is above $u$ for any fixed $s$. The Lipschitz property of $H$ in its last argument implies that, for all $x, u, u^{\prime}$,

$$
-K u^{\prime} \leq H(x, u, 0)-H\left(x, u, u^{\prime}\right) .
$$

From the definition of $u$, this implies that

$$
u^{\prime \prime}(x)+H\left(x, u(x), u^{\prime}(x)\right) \leq-\varepsilon<0
$$

for all $x$. This implies that $w$, the solution to the IVP, lies above $u$, by the following argument. At $x=0$, $u$ and $w$ have the same starting values and slopes, but $u$ has a lower second derivative, by at least $\varepsilon$, which implies that $u^{\prime}<w^{\prime}$ in a right neighborhood of 0 . We will show that $u^{\prime}<w^{\prime}$ for all $x$ in $(0, \bar{x}]$ and, therefore, that $u<w$ on that domain. Suppose by contradiction that there exists an $x>0$ such that $u^{\prime}(x)=w^{\prime}(x)$, and let $\tilde{x}$ be the first such point. Necessarily, $u(\tilde{x})<w(\tilde{x})$. Moreover, we have

$$
u^{\prime \prime}(\tilde{x})<-H\left(\tilde{x}, u(\tilde{x}), u^{\prime}(\tilde{x})\right) \leq-H\left(\tilde{x}, w(\tilde{x}), w^{\prime}(\tilde{x})\right)=w^{\prime \prime}(\tilde{x}),
$$

where the second inequality is guaranteed by Condition 2. This contradicts the fact that $u^{\prime}$ crosses $w^{\prime}$ from below at $\tilde{x}$.

We can finally prove Proposition 1. Let

$$
B=\left\{(x, v) \mid b_{1}(x)=v \text { or } b_{2}(x)=v\right\} \cup\left[\left(\bar{x}, b_{1}(\bar{x})\right),\left(\bar{x}, b_{2}(\bar{x})\right)\right] \subset \mathbb{R}^{2} .
$$

$B$ consists of the graph of the functions $b_{1}$ and $b_{2}$ on $\mathcal{X}$, along with the vertical segment joining the endpoints of these graphs at $\bar{x}$. We also define the function $\mathcal{H}:\left[s_{1}, s_{2}\right] \rightarrow \mathbb{R}^{2}$ as the last hitting point of $B$ for the solution of the IVP with slope $s$. This function is clearly well defined: if a solution does not cross $b_{1}$ or $b_{2}$ before $\bar{x}$, it has to hit the vertical segment joining $b_{1}(\bar{x})$ and $b_{2}(\bar{x})$. From Lemma $7 \mathcal{H}(s)$ is on the graph of $b_{2}$ for $s$ large and on the graph of $b_{1}$ for $s$ small (for example, (32) shows that, for $s$ large enough, $u$ cannot cross $b_{2}$ again after hitting it once). Moreover, $\mathcal{H}$ cannot jump from the graph of $b_{2}$ to the graph of $b_{1}$ as $s$ changes, because Lemma 6 implies, for example, that if $w$ crosses $b_{2}$, it stays above $b_{2}$ for all $x$ beyond the crossing point 81 and hence cannot hit $b_{1}$. Therefore, $\mathcal{H}$ must connect the upper and lower bounds of $B$ as $s$ goes down. Finally, Lemma 5 implies that $\mathcal{H}$ is continuous at any point $s$ for which $\mathcal{H}(s)$ lies on the vertical segment. This shows that $\mathcal{H}(s)$ must take all values on that segment as it connects the graphs of $b_{2}$ and $b_{1}$. Since $(\bar{x}, \bar{v})$ belongs to that segment, this proves existence of a solution that solves the BVP.

\footnotetext{
${ }^{81}$ The proof of this result is similar to the proof that $w$ stays above $u$ in Lemma 7 showing that $w^{\prime} \geq b_{2}^{\prime}$ after the crossing point, and exploits the inequality $b_{2}^{\prime \prime}+H\left(x, b_{2}, b_{2}^{\prime}\right)<0$.
} 


\section{B.3 Proof of Proposition 1: Unbounded Domain}

We now prove Proposition 1 when $\mathcal{X}$ is unbounded, so that $\underline{x}=-\infty$ and/or $\bar{x}=+\infty$. Precisely, we establish the existence of a function $v$ which satisfies

$$
w^{\prime \prime}=-H\left(x, w, w^{\prime}\right)
$$

and $|w(x)| \leq K_{v}(1+|x|)$ on $\mathcal{X}$, where $K_{v}$ is a positive constant. The arguments of this section are based on Schrader (1969). For expositional simplicity we focus on the case in which $\mathcal{X}=\mathbb{R}$. The case in which either $\underline{x}$ or $\bar{x}$ is finite follows easily from that argument.

Throughout the argument we fix a pair $K_{1}, K_{2}$ of constants that satisfy Condition 3 , and let $b_{1}, b_{2}$ denote the bounds constructed from these constants in Lemma 6. From Section B.2, we know that the BVP will have a unique $C^{2}$ solution for any finite interval $[\underline{\chi}, \bar{\chi}]$ and boundary conditions $w(\underline{\chi})=\underline{v}$ and $w(\bar{\chi})=\bar{v}$ that are contained between $b_{1}$ and $b_{2}$. Furthermore, we know that the solution satisfies $-K_{v}(1+|x|) \leq$ $w(x) \leq K_{v}(1+|x|)$ on $[\chi, \bar{\chi}]$, where $K_{v}=\max \left\{K_{1}, K_{2}\right\}$ does not depend on the particular interval chosen.

We define a sequence of boundary value problems such that Equation $(33)$ holds on $\left[\underline{x}_{n}, \bar{x}_{n}\right]$ and with boundary conditions $w\left(\underline{x}_{n}\right)=\underline{v}_{n}$ and $w\left(\bar{x}_{n}\right)=\bar{v}_{n}$ for some values $\underline{v}_{n}, \bar{v}_{n}$ in $\left(b_{1}\left(\underline{x}_{n}\right), b_{2}\left(\underline{x}_{n}\right)\right)$ and $\left(b_{1}\left(\bar{x}_{n}\right), b_{2}\left(\bar{x}_{n}\right)\right)$, respectively, and let $\underline{x}_{n}$ and $\bar{x}_{n}$ tend to $-\infty$ or $+\infty$, respectively.

Let $w_{n}$ denote the solution to the $n^{\text {th }}$ BVP. In the following, we use the Arzelà-Ascoli theorem and show that this procedure indeed yields a solution. For this, we need to prove that the derivatives of $w_{n}$ are equicontinuous, using the following lemma (Hartman 2002, p. 428).

LEMMA 8 Let $\phi$ be a nonnegative, continuous function on $\mathbb{R}_{+}$such that

$$
\int_{0}^{\infty} \frac{s}{\phi(s)} d s=\infty
$$

and let $R, \tilde{x}$ denote two strictly positive constants. Then, there exists a number $M$ such that if $w(x)$ is $C^{2}$ on $[0, \bar{x}]$ with $\bar{x}>\tilde{x}$ and satisfies $|w(x)| \leq R$ and $\left|w^{\prime \prime}(x)\right| \leq \phi\left(\left|w^{\prime}(x)\right|\right)$, then $\left|w^{\prime}(x)\right| \leq M$ on $[0, \bar{x}]$. The constant $M$ depends only on $R, \phi$ and $\tilde{x}$.

For any bounded domain $\mathcal{X}_{0}=[\underline{\chi}, \bar{\chi}]$ and any solution $w$ to the BVP on that domain with end values between $b_{1}$ and $b_{2}$, we have

$$
\left|w^{\prime \prime}(x)\right|=\mid H\left(x, w(x), w^{\prime}(x)|\leq| H(x, w(x), 0)|+K| w^{\prime}(x)|\leq \bar{K}+K| w^{\prime}(x) \mid\right.
$$

where $K$ is the Lipschitz constant of $H$ over $\mathcal{X}_{0}$ and where the constant $\bar{K}$ comes from the boundedness of $w$ (which is contained between $b_{1}$ and $b_{2}$ ) and continuity of $H(\cdot, \cdot, 0)$ on the compact domain $\mathcal{X}_{0}$.

Since $\phi(x)=\bar{K}+K x$ satisfies (34) and $w$ is bounded by the functions $b_{1}$ and $b_{2}$, Lemma 8 implies that each $w_{n}^{\prime}$ is bounded on the compact domain $\mathcal{X}_{0}$, and that the bound is uniform over $n$ M2 Moreover, (35) implies that the second derivatives of $w_{n}$ are also uniformly bounded on $\mathcal{X}_{0}$.

We now use the following diagonalization procedure. Consider a finite domain $\left[\underline{x}_{1}, \bar{x}_{1}\right]$. We have seen that the functions $w_{n}, w_{n}^{\prime}$ and $w_{n}^{\prime \prime}$ are bounded on $\left[\underline{x}_{1}, \bar{x}_{1}\right]$, uniformly in $n$. By Arzelà-Ascoli's theorem, there

\footnotetext{
${ }^{82}$ More precisely, we can apply the Lemma to the function $\bar{w}(x)=w(x-\underline{\chi})$, so as to have the 0 origin that is assumed in the Lemma.
} 
exists a subsequence such that $w_{n}$ converges uniformly to a $C^{1}$ function $\tilde{w}_{1}$ on $\left[\underline{x}_{1}, \bar{x}_{1}\right]{ }^{83}$ Moreover, the second derivatives $\left\{w_{n}^{\prime \prime}\right\}_{n \in \mathbb{N}}$ are also equicontinuous, because they satisfy $w_{n}^{\prime \prime}(x)=-H\left(x, w_{n}(x), w_{n}^{\prime}(x)\right)$ with $H$ continuous and $w_{n}$ and $w_{n}^{\prime}$ equicontinuous. This implies that there is a subsequence of $w_{n}$ that converges uniformly to a $C^{2}$ function $\tilde{w}_{1}$ on $[\underline{x}, \bar{x}]$. This also implies that the limit satisfies $\tilde{w}_{1}^{\prime \prime}(x)=-H\left(x, \tilde{w}_{1}(x), \tilde{w}_{1}^{\prime}(x)\right)$. By construction, $b_{1}(x) \leq w_{n}(x) \leq b_{2}(x)$ on $\left[\underline{x}_{1}, \bar{x}_{1}\right]$ and, therefore, $\tilde{w}_{1}$ is also contained between $b_{1}$ and $b_{2}$.

To conclude, take the finite domain $\left[\underline{x}_{2}, \bar{x}_{2}\right] \supset\left[\underline{x}_{1}, \bar{x}_{1}\right]$. Iterating the last argument ${ }^{84}$ there exists a subsequence of the first subsequence for which $w_{n}$ converges uniformly to a limit function $\tilde{w}_{2}$ on $\left[\underline{x}_{2}, \bar{x}_{2}\right]$. The functions $\tilde{w}_{1}$ and $\tilde{w}_{2}$ are equal on $\left[\underline{x}_{1}, \bar{x}_{1}\right]$. Proceeding iteratively, we can cover the entire domain $\mathcal{X}$. The function $w$ defined by $w(x)=\tilde{w}_{k}(x)$ for $x \in\left[\underline{x}_{k}, \bar{x}_{k}\right] \backslash\left[\underline{x}_{k-1}, \bar{x}_{k-1}\right]$, solves the BVP and is bounded by $b_{1}$ and $b_{2}$. It thus also satisfies $|w(x)| \leq K_{v}(1+|x|)$.

\section{Extension of Berge's Maximum Theorem to Non-Compact Do- mains}

Proposition 8 Suppose that $h: \mathcal{X} \times \mathcal{K} \rightarrow \mathbb{R}$ is uniformly continuous and $\mathcal{X}$ and $\mathcal{K}$ are metric spaces. Then, the function $H: \mathcal{X} \rightarrow \mathbb{R}$ defined by

$$
H(x)=\sup _{a \in \mathcal{K}} h(x, a)
$$

is continuous.

Proof. Aliprantis and Border (2006), Lemma 17.29, p. 569, shows that $H$ is lower semicontinuous. It remains to prove that it is upper semicontinuous. For $\alpha \in \mathbb{R}$, consider the strict lower level set $X_{\alpha}=\{x: H(x)<\alpha\}$. We need to prove that $X_{\alpha}$ is an open set for any $\alpha$. By construction, for any $x_{0} \in X_{\alpha}, h\left(x_{0}, a\right)<\alpha$ for all $a$. By uniform continuity one may choose, for any $\varepsilon>0$, a $\delta>0$ small enough that for any $x^{\prime}$ with $\left|x^{\prime}-x_{0}\right|<\delta$, we have

$$
\left|\sup _{\alpha \in \mathcal{K}} h\left(x^{\prime}, a\right)-\sup _{\alpha \in \mathcal{K}} h\left(x_{0}, a\right)\right| \leq \sup _{a \in \mathcal{K}}\left|h\left(x^{\prime}, a\right)-h\left(x_{0}, a\right)\right|<\varepsilon,
$$

where the first inequality is shown similarly to Footnote $26{ }^{85}$ Therefore, $x^{\prime} \in X_{\alpha}$ for $\delta$ sufficiently small and the set $X_{\alpha}$ is open.

\section{Optimal Growth Extension: Vanishing Volatility}

This appendix reconsiders the growth example of Section 3.1 and explicitly allows the volatility to reach zero at the origin, illustrating how the techniques used in Appendix B.1 can be extended for this case.

\footnotetext{
${ }^{83}$ More precisely, we use the following version: any sequence of $C^{1}$ functions that have equicontinuous and uniformly bounded derivatives, and are uniformly bounded at one point, has a subsequence that converges uniformly to a $C^{1}$ function. Here, equicontinuity of the derivatives is guaranteed by the uniform bound on the second derivative.

${ }^{84}$ Note that the bounds for the domains $\left[\underline{x}_{2}, \bar{x}_{2}\right]$ and $\left[\underline{x}_{1}, \bar{x}_{1}\right]$ are different. However, since we are fixing the domain, we are still able to obtain a convergent subsequence.

${ }^{85}$ There, the inequality was shown when the supremum was reached. It is straightforward to extend the proof when it is not.
} 
The decision maker solves

$$
v\left(x_{0}\right)=\sup _{A \in \mathcal{A}} E\left[\int_{0}^{\tau} e^{-r t} f\left(X_{t}^{A}, A_{t}\right) d t\right]
$$

subject to

$$
d X_{t}^{A}=\mu\left(X_{t}^{A}, A_{t}\right) d t+\sigma\left(X_{t}^{A}, A_{t}\right) d B_{t}
$$

and $X_{0}^{A}=x_{0}>0$. The state space is given by $\mathcal{X}=[0, \infty)$ and the state $X_{t}^{A}=0$ is absorbing, so that $X_{s}^{A}=0$ for all $s>\tau$, where $\tau=\inf \left\{t \geq 0: X_{t}^{A}=0\right\}$. We normalize $\max _{a \in \mathcal{K}(0)} f(0, a)$ to 0 and $\operatorname{relax}$ Assumptions $1-3$, so that the diffusion coefficient $\sigma(x, a)$ can reach zero at $x=0$ and $f, \mu$, and $\sigma$ do not have to satisfy the Lipschitz assumptions at $x=0$.

Assumption 1' $A_{t} \in \mathcal{K}\left(X_{t}^{A}\right)$ for all $t$, where $\left.\mathcal{K}(x)=[0, K(x))\right]$ for some nonnegative continuous function $K(x)$.

Assumption 2' For any $[\underline{x}, \bar{x}]$ with $\underline{x}>0$, there exists a constant $K>0$ such that for all $\left(x, x^{\prime}, a\right)$ satisfying $x, x^{\prime} \in[\underline{x}, \bar{x}]$ and $a \in \mathcal{K}(x) \cap \mathcal{K}\left(x^{\prime}\right)$,

$$
\left|\mu(x, a)-\mu\left(x^{\prime}, a\right)\right|+\left|\sigma(x, a)-\sigma\left(x^{\prime}, a\right)\right|+\left|f(x, a)-f\left(x^{\prime}, a\right)\right| \leq K\left|x-x^{\prime}\right|,
$$

and the functions $\mu, \sigma$, and $f$ are jointly continuous in $(x, a)$.

Assumption 3' There exist constants $K_{1}^{\mu}, K_{2}^{\mu}, K^{\sigma}$, and $K^{f}$ such that $K_{2}^{\mu}<r,|\mu(x, a)| \leq K_{1}^{\mu}+K_{2}^{\mu}|x|$, $|\sigma(x, a)| \leq K^{\sigma}(1+|x|)$, and $|f(x, a)| \leq K^{f}(1+|x|)$ for all $(x, a)$ such that $x \in \mathcal{X}$ and $a \in \mathcal{K}(x)$. Further, for all $\underline{x}>0$, there exists a constant $\underline{\sigma}_{x}>0$ such that for all $x \in[\underline{x}, \infty), \min _{a \in \mathcal{K}(x)} \sigma(x, a) \geq \underline{\sigma}_{x}$.

Proposition 9 Let $\sigma_{0}(x)=\min _{a \in \mathcal{K}(x)} \sigma(x, a)$. Suppose, in addition to Assumptions 1'-3', $\sigma_{0}^{-2}(x) \in$ $L^{P}([0, \bar{x}])$ for some $P>1$ and $\bar{x}>086$ Then, the HJB equation

$$
r v(x)=\max _{a \in \mathcal{K}(x)} f(x, a)+v^{\prime}(x) \mu(x, a)+\frac{1}{2} \sigma^{2}(x, a) v^{\prime \prime}(x)
$$

with the boundary condition $v(0)=0$ has a unique solution which is twice differentiable on $(0, \infty)$ and continuous at $x=0$. This solution equals the decision maker's value function on $[0, \infty)$.

The proof is a modification of the argument in Appendix B.3 and relies on the following extension of Nagumo's condition, due to Kiguradze (1967).

Lemma 9 Let $E=[a, b] \times[-r, r] \times \mathbb{R}$ for some $a<b$ and $r>0$ and let $P, Q \in[1, \infty]$ be such that $\frac{1}{P}+\frac{1}{Q}=1$. If for some continuous functions $H: E \rightarrow \mathbb{R}$ and $\phi: \mathbb{R}_{+} \rightarrow \mathbb{R}_{++}$and some function $\psi:[a, b] \rightarrow \mathbb{R}_{+}$with $\psi \in L^{P}([a, b])$ we have

$$
\int_{0}^{\infty} \frac{s^{\frac{1}{Q}}}{\phi(s)} d s=\infty
$$

and

$$
|H(x, p, q)| \leq \psi(x) \phi(|q|)
$$

\footnotetext{
${ }^{86}$ For any interval $I$ of $\mathbb{R}, \phi \in L^{P}(I)$ if and only if $\left(\int_{I}(|\phi(x)|)^{P} d x\right)^{\frac{1}{P}}<\infty$, where the integral is taken with respect to the Lebesgue measure.
} 
then there exists a number $M>0$ such that for any solution to

$$
w^{\prime \prime}(x)=H\left(x, w(x), w^{\prime}(x)\right)
$$

on $[a, b]$ with $|w(x)| \leq r$, we have

$$
\left|w^{\prime}(x)\right|<M
$$

We again consider a sequence of domains $\left[\underline{x}_{n}, \bar{x}_{n}\right]$ with $\underline{x}_{n} \rightarrow 0$ and $\bar{x}_{n} \rightarrow \infty$ as $n \rightarrow \infty$ and the associated boundary value problems with boundary conditions $w\left(\underline{x}_{n}\right)=\underline{v}_{n}$ and $w\left(\bar{x}_{n}\right)=\bar{v}_{n}$ and solutions $w_{n}(x)$. We choose $\underline{v}_{n} \rightarrow 0$ and $\bar{v}_{n}$ as in Appendix B.3. On any domain $[\underline{x}, \bar{x}]$ with $0<\underline{x}<\bar{x}<\infty$,

$$
H(x, p, q)=\max _{a \in \mathcal{K}(x)} \frac{1}{\sigma^{2}(x, a)}(-r p+f(x, a)+\mu(x, a) q)
$$

satisfies Conditions 1-3 used in the proof of Proposition 2 and a subsequence of $w_{n}(x)$ converges to the twice differentiable function $w(x)$.

It remains to show that the function $w(x)$ constructed by this method is continuous at zero. Since

$$
\begin{aligned}
|H(x, p, q)| & \leq\left|\frac{1}{\sigma_{0}^{2}(x)}\right| \max _{a \in \mathcal{K}(x)}(r|p|+|f(x, a)|+|\mu(x, a)||q|) \\
& \leq\left|\frac{1}{\sigma_{0}^{2}(x)}\right|(\bar{K}+K|q|),
\end{aligned}
$$

taking $\psi(x)=\frac{1}{\sigma_{0}^{2}(x)}$ and $\phi(x)=\bar{K}+K s$ in Lemma 9 implies that on any finite interval $[0, \bar{x}], w_{n}^{\prime}(x)$ is bounded whenever $w_{n}(x)$ is bounded 87 On any such $[0, \bar{x}]$, the sequence $w_{n}(x)$ is thus equicontinuous and a subsequence converges uniformly to $w(x)$ by the Arzelà-Ascoli theorem. Therefore, $w(x)$ is continuous at zero. Since $w(x)$ is twice differentiable except at zero, the verification argument in Lemmas 3 and 4 applies without modification.

EXAMPLE 1 Take a version of the neoclassical growth model in which the capital stock evolves as

$$
d k_{t}=\left(k_{t}^{\alpha}-\delta k_{t}-c_{t}\right) d t+\sigma k_{t}^{\rho} d B_{t}
$$

with initial condition $k_{0}>0$ and $0<\rho<\frac{1}{2}$. Here, $\delta>0$ is the depreciation rate and $k^{\alpha}$ with $\alpha \in(0,1)$ is the production function. With CRRA utility $u(c)=\frac{c^{(1-\gamma)}-1}{1-\gamma}$ for $\gamma \in(0,1)$ and feasible consumption set $\mathcal{K}(k)=[0, M k]$ for $M>0$ chosen sufficiently large, this problem satisfies the assumptions of Proposition 9.

\section{E Results for the Multi-Armed Bandit Application}

Lemma 10 The value function $v$ is convex in the belief $x$.

Proof. The argument is standard and replicated for completeness. For any admissible control process $A$, we have

$$
v(x, A)=E\left[\int_{0}^{\infty} e^{-r t} \pi\left(A_{t}, \tilde{\theta}\right) d t \mid x\right]
$$

\footnotetext{
${ }^{87}$ Note that $\int_{0}^{\infty} \frac{s^{\frac{1}{q}}}{\bar{K}+K s} d s=\infty$ for any $1 \leq q<\infty$.
} 
By definition, any admissible process $A$ is adapted to the observation filtration, which, conditional on the true state $\tilde{\theta}$ does not depend on the probability $x$. Therefore, we have

$$
v(x, A)=x E\left[\int_{0}^{\infty} e^{-r t} \pi\left(A_{t}, \theta_{H}\right) d t \mid \theta_{H}\right]+(1-x) E\left[\int_{0}^{\infty} e^{-r t} \pi\left(A_{t}, \theta_{L}\right) d t \mid \theta_{L}\right],
$$

which shows that $v(x, A)$ is linear in $x$. For $x_{1}<x_{2}$ and $\lambda \in(0,1)$, let $x=\lambda x_{1}+\left(1-\lambda x_{2}\right)$. We have, letting $A^{*}$ denote an optimal process given the initial belief $x{ }^{88}$

$$
v(x)=v\left(x, A^{*}\right)=\lambda v\left(x_{1}, A^{*}\right)+(1-\lambda) v\left(x_{2}, A^{*}\right) \leq \lambda v\left(x_{1}\right)+(1-\lambda) v\left(x_{2}\right),
$$

where the second equality comes from the linearity of $v(\cdot, A)$ in $x$, and the inequality comes from the definition of the $v\left(x_{i}\right)$ 's.

Proposition 10 With finitely many arms, the are finitely many cutoffs, and the number of cutoffs is bounded by twice the number of arms.

Proof. From Theorem 1 the value function solves everywhere the HJB equation, which may be rewritten as

$$
r v(x)=\max _{i \in \mathcal{K}}\left\{x \Delta f(i)+f_{L}(i)+v^{\prime \prime}(x) \frac{1}{2}(x(1-x))^{2} \xi^{2}(i)\right\}
$$

where $\Delta f(i)=f_{H}(i)-f_{L}(i)$. If $v^{\prime \prime}(x)=0$ for some $x \in(0,1)$, then $v(x)=\frac{x \Delta f(i)+f_{L}(i)}{r}$ for some $i$, which means that playing $i$ forever is optimal and there is no cutoff. Thus, we focus on the case $v^{\prime \prime}(x)>0$ for all $x \in(0,1)$.

Rewriting the HJB equation, we have

$$
v^{\prime \prime}(x)=\min _{i \in \mathcal{K}} \frac{r v(x)-x \Delta f(i)-f_{L}(i)}{\frac{1}{2}(x(1-x))^{2} \xi^{2}(i)} .
$$

Moreover, letting

$$
w_{i}(x)=\frac{r v(x)-x \Delta f(i)-f_{L}(i)}{\xi^{2}(i)},
$$

Arm $i$ maximizes 36 if and only if $w_{i}(x) \leq w_{j}(x)$ for all $j$ in $\mathcal{K}$.

We have

$$
\left(w_{i}-w_{j}\right)^{\prime \prime}(x)=r v^{\prime \prime}(x)\left(\frac{\xi^{2}(j)-\xi^{2}(i)}{\xi^{2}(i) \xi^{2}(j)}\right) .
$$

Lemma 10 therefore implies that either $w_{i}-w_{j}$ or $w_{j}-w_{i}$ is convex. If $w_{i}-w_{j}$ is linear, either the arms are identical, in which case they can be treated as a single arm, or $w_{i}-w_{j}$ crosses zero at most once. Otherwise, $w_{i}-w_{j}$ is strictly convex or strictly concave, since $v^{\prime \prime}$ is strictly positive, and crosses zero at most twice. Since the number of arms is finite, this implies that the set $\mathcal{Z}_{i}=\left\{x: w_{i}(x)>w_{j}(x) \quad \forall j \neq i\right\}$ consists of finitely many open intervals, and that the set $\mathcal{Z}$ of $x \in[0,1]$ such that $\arg \max _{i}\left\{w_{i}(x)\right\}$ is not a singleton is finite. This shows that any selection of maximizers of the HJB equation has finitely many switches, yielding a volatility that has bounded variation. Theorem 2 then implies that such selection is optimal. It also implies that the (essentially unique) optimal strategy has finitely many cutoffs. Because any two arms can

\footnotetext{
${ }^{88}$ We do not need to assume the existence of an optimum: the argument is easily adapted by taking a sequence of controls $A_{n}$ such that $v\left(x, A_{n}\right)$ converges to $v(x)$ as $n \rightarrow \infty$.
} 
only change dominance twice, this also shows that the number of switching points is bounded by twice the number of arms: if an arm is strictly optimal in two disjoint regions, then any arm that is strictly optimal in a region contained between these two regions can only be optimal within these two regions. The result then follows by an easy induction argument.

Proposition 11 A given arm may be used over disjoint intervals of beliefs.

Proof. Consider a four-armed setting with the following characteristics. Arms 1 and 2 have symmetric payoffs around the belief $1 / 2: f_{1}(x)=f_{2}(1-x)$, with $f_{1}(0)>f_{1}(1)$, and have the same low signal-to-noise ratio $\xi(1)=\xi(2)=\varepsilon>0$. The payoffs $f_{3}$ and $f_{4}$ are independent 89 of $x$, with $f_{1}(0)>f_{3}>f_{4}$ and $\min \{\xi(3), \xi(4)\}>\varepsilon$.

Notice that, for extreme beliefs, either Arm 1 or 2 is used. Moreover, because Arm 1 is optimal for very low beliefs whereas Arm 2 is optimal for very high ones, the value of information is strictly positive in this problem.

It suffices to show that Arms 3 and 4 are both chosen on a positive domain. By symmetry, this will imply that one arm is chosen on both sides (the one that is not used at $x=1 / 2$ ) ${ }^{90}$ Consider, first, the case in which only Arms 1,2, and 3 are available. Because Arms 1 and 2 have low signal to noise ratio, using Arm 3 is optimal for intermediate beliefs, provided that $f_{3}$ is high enough (but still below $f_{1}(0)$ ). We now add in Arm 4, which has a low payoff. We will gradually increase its signal to noise ratio, $\xi(4)$. For $\xi(4)<\xi(3)$, Arm 4 is dominated by Arm 3 both in terms of payoff and informativeness, and thus not used at all. For $\xi(4)$ arbitrarily high, using Arm 4 reveals the state of the world almost instantaneously, which is clearly optimal for intermediate beliefs. Therefore, there must exist a threshold $\xi_{4}^{*}>\xi(3)$ above which it becomes strictly optimal to use Arm 4 around some belief $x^{*}$. At $\xi_{4}^{*}, v_{4}-v_{3}$ has a local maximum at $x^{*}$, which has to be in the interior of $[0,1]$. From the proof of Proposition 10, this implies that $v_{4}-v_{3}$ is strictly concave and that $x^{*}$ is the unique global maximum. This implies that $v_{3}(x)>v_{4}(x)$ for all $x \neq x^{*}$ and, since it is always optimal to use either Arm 3 or Arm 4 on an interval of strictly positive measure, this implies that $v_{3}\left(x^{\prime}\right)>\max \left\{v_{1}\left(x^{\prime}\right), v_{2}\left(x^{\prime}\right), v_{4}\left(x^{\prime}\right)\right\}$ on some interval $\mathcal{Z}^{\prime}$ of positive measure. By increasing $\xi(4)$ slightly above $\xi_{4}^{*}$, Arm 4 becomes strictly optimal around $x^{*}$ (by definition of $x^{*}$ ), while Arm 3 remains strictly optimal on $\mathcal{Z}^{\prime}$, which proves the claim.

\section{F Proofs of Section 5 (Smooth Pasting)}

Lemma 11 Consider $v_{\tilde{s}}$ and $v_{s}$, two solutions to the IVP with starting slopes $\tilde{s}>s$ on an interval $\left[x_{1}, x_{2}\right]$ which both satisfy $v_{\tilde{s}}\left(x_{1}\right)=v_{s}\left(x_{1}\right)=v_{1}$. Then, $v_{\tilde{s}}(x)>v_{s}(x)$ for all $x \in\left(x_{1}, x_{2}\right]$.

Proof. Let $\hat{x}=\inf \left\{x: v_{\tilde{s}}^{\prime}(x) \leq v_{s}^{\prime}(x)\right\}$. Note that $\hat{x}>\underline{x}$ because $v_{\tilde{s}}^{\prime}(\underline{x})>v_{s}^{\prime}(\underline{x})$ and both $v_{\tilde{s}}$ and $v_{s}$ are

\footnotetext{
${ }^{89}$ Thus, for simplicity, Arms 3 and 4 are informative despite having state-independent payoffs. It is easy to slightly perturb those payoffs to introduce payoff dependence and preserve the qualitative shape of the choice intervals.

${ }^{90}$ If $x=1 / 2$ was a cutoff, then both Arms 3 and 4 are used on each side of $x=1 / 2$, which also proves the proposition.
} 
$C^{2}$. By construction, $v_{\tilde{s}}(\hat{x})>v_{s}(\hat{x})$. Since both solutions satisfy the equation

$$
v^{\prime \prime}(x)+\frac{1}{\frac{1}{2} \sigma(x)^{2}}\left(-r v(x)+f(x)+\mu(x) v^{\prime}(x)\right)=0
$$

we have

$$
\begin{aligned}
v_{\tilde{s}}^{\prime \prime}(\hat{x}) & =\frac{1}{\frac{1}{2} \sigma(\hat{x})^{2}}\left(r v_{\tilde{s}}(\hat{x})+f(\hat{x})+\mu(\hat{x}) v_{\tilde{s}}^{\prime}(\hat{x})\right) \\
& >\frac{1}{\frac{1}{2} \sigma(\hat{x})^{2}}\left(r v_{s}(\hat{x})+f(\hat{x})+\mu(\hat{x}) v_{s}^{\prime}(\hat{x})\right)=v_{s}^{\prime \prime}(\hat{x})
\end{aligned}
$$

Since $v_{\tilde{s}}^{\prime}(x)$ must hit $v_{s}^{\prime}(x)$ from above as $x$ reaches $\hat{x}$, we obtain a contradiction.

\section{F.1 End of the proof of Theorem 4}

To conclude the proof, we need to show the result when $x$ is such that $v(x)=g(x)$, but $x$ is an accumulation point of stopping and continuation regions, on either its right side or its left side, or both.

Without loss of generality, we set $x=0$ and prove that $v_{r}(0)=g^{\prime}(0)$, where $v_{r}$ is the right derivative of $v$ at 0 . We wish to show that $\lim _{\eta \downarrow 0}(v(\eta)-v(0)) / \eta$ converges to $g^{\prime}(0)$. Consider any $\eta>0$. The difference $v(\eta)-v(0)$ is either equal to $g(\eta)-g(0)$, if $\eta$ belongs to $\mathcal{Y}$, or else $\eta$ belongs to some interval $\mathcal{Z}_{i}$ close to 0 . Let $y$ denote the lower bound of $\mathcal{Z}_{i}$. By twice differentiability of $v$ on $(y, \eta)$, and because the right derivative of $v$ at $y$ is equal to $g^{\prime}(y)$, we have $v(\eta)=v(y)+g^{\prime}(y)(\eta-y)+\frac{1}{2} v_{r}^{\prime \prime}\left(z_{1}\right)(\eta-y)^{2}$ for some $z_{1} \in(y, \eta)$. Since $v(y)=g(y)$, we have $g(\eta)=v(y)+g^{\prime}\left(z_{2}\right)(\eta-y)$ for some $z_{2} \in(y, \eta)$. Therefore, $v(\eta)=g(\eta)+\left(g^{\prime}(y)-g^{\prime}\left(z_{2}\right)\right)(\eta-y)+\frac{1}{2} v_{r}^{\prime \prime}\left(z_{1}\right)(\eta-y)^{2}$, and

$$
\frac{v(\eta)-v(0)}{\eta}=\frac{g(\eta)-g(0)}{\eta}+1_{\eta \notin \mathcal{Y}} \frac{\eta-y}{\eta}\left(g^{\prime}(y)-g^{\prime}\left(z_{2}\right)+\frac{1}{2} v_{r}^{\prime \prime}\left(z_{1}\right)(\eta-y)\right) .
$$

Taking the limit as $\eta$ goes to zero yields the result, if we can show that $g^{\prime}(y)-g^{\prime}\left(z_{2}\right)+\frac{1}{2} v_{r}^{\prime \prime}\left(z_{1}\right)(\eta-y)$ converges to zero as $\eta \rightarrow 0$. The first two terms cancel each other in the limit, as they both converge to $g^{\prime}(0)$ (since $g$ is $C^{1}$ ). The last term converges to 0 if we can show that $v^{\prime \prime}(\cdot)$ is uniformly bounded on all the intervals $\mathcal{Z}_{i}$ in a neighborhood of 0 . This uniform bound is guaranteed by Lemma 8 (Section B.3), which guarantees a uniform upper bound on $\left|v^{\prime}\right|$ and on $\left.\left|v^{\prime \prime}\right|\right|^{91}$

Continuity of $v^{\prime}$ is shown by a similar argument. For any $\varepsilon>0$, there exists $\bar{\eta}(\varepsilon)$ such that $\left|g^{\prime}(\eta)-g^{\prime}(0)\right| \leq \varepsilon / 2$ for all $\eta \leq \bar{\eta}(\varepsilon)$. As was mentioned earlier, $v^{\prime \prime}$ is uniformly bounded on the interval $[x, x+\bar{\eta}(\varepsilon)] \cap \cup_{i \in \mathcal{I}} \mathcal{Z}_{i}$, by some constant $M$. Let $\eta(\varepsilon)=\min \{\bar{\eta}(\varepsilon), \varepsilon / M\}$. Consider any $\eta<\eta(\varepsilon)$. If $\eta \in \mathcal{Y}$, then $v^{\prime}(\eta)=g^{\prime}(\eta)$ and $\left|v^{\prime}(\eta)-v^{\prime}(0)\right|<\varepsilon / 2$. Otherwise we have, using the variable $y$ introduced earlier in the proof,

$$
v^{\prime}(\eta)=v^{\prime}(y)+v^{\prime \prime}\left(z_{3}\right)(\eta-y)=g^{\prime}(y)+v^{\prime \prime}\left(z_{3}\right)(\eta-y),
$$

\footnotetext{
${ }^{91}$ More precisely, the solution $w_{i}$ to the BVP on $\mathcal{Z}_{i}$ with endpoints $\left[\underline{x}_{i}, \bar{x}_{i}\right]$ can be extended to a solution on the domain $\mathcal{Z}_{i}^{\prime}=\left[\underline{x}_{i}, \max \left\{\underline{x}_{i}+\tilde{x}, \bar{x}_{i}\right\}\right]$, for some $\tilde{x}>0$ that is independent of $i$, by considering the IVP with domain $\mathcal{Z}_{i}^{\prime}$ and keeping the same initial value and slope at $\underline{x}_{i}$. That solution has uniformly bounded first derivative, by Lemma 8 and hence also a uniformly bounded second derivative (see (35). The uniform bounds do not depend on $i$ : they only depend on $\tilde{x}$, an upper bound on $v$, and the fact that $\left|w^{\prime \prime}(x)\right|=\left|\frac{1}{\frac{1}{2} \sigma(x)^{2}}\left(-r w(x)+f(x)+\mu(x) w^{\prime}(x)\right)\right| \leq K_{1}+K_{2}\left|w^{\prime}(x)\right|$ for some constants $K_{1}, K_{2}$.
} 
for some $z_{3} \in(y, \eta)$. This implies that

$$
\left|v^{\prime}(\eta)-v^{\prime}(0)\right|<\left|g^{\prime}(y)-g^{\prime}(0)\right|+M \eta<\varepsilon .
$$

Proceeding similarly to the left of $x$, we conclude that $v^{\prime}$ is continuous at $x$. 


\section{References}

Aliprantis, C., Border, K. (2006) Infinite Dimensional Analysis, Second Edition, Springer-Verlag.

Ambrosio, L., Dal Maso, G. (1990) "A General Chain Rule for Distributional Derivatives," Proceedings of the American Mathematical Society, Vol. 108, No. 3, pp. 691-702.

Amir, R., De Castro, L. (2013) "Nash Equilibrium in Games with Quasi-Monotonic Best-Responses," Working Paper.

Bailey, P. (1968) Nonlinear Two Point Boundary Value Problems, Academic Press.

Barlow, M. (1982) "One Dimensional Stochastic Differential Equations with No Strong Solution," Journal of the London Mathematical Society, Vol s2-26, pp. 335-347.

Bebernes, J. W. (1963) "A Subfunction Approach to a Boundary Value Problem for Ordinary Differential Equations," Pacific Journal of Mathematics, Vol. 13(4), pp. 1053-1066.

Benveniste, L. M., Scheinkman, J. A. (1979) "On the Differentiability of the Value Function in Dynamic Models of Economics," Econometrica, Vol. 47(3), pp. 727-732.

Bergemann, D., VÄLimÄki, J. (1997) "Market-Diffusion with Two-Sided Learning," RAND Journal of Economics, Vol. 28, No. 4, pp. 773-795.

Bergemann, D., VÄLimäki, J. (2000) "Experimentation in Markets," Review of Economic Studies, Vol. 67, pp. 213-234.

Bolton, P., Harris, C. (1999) "Strategic Experimentation," Econometrica, Vol. 67, No. 2, pp. 349-374.

Borkar, V. (2005) "Controlled Diffusion Processes," Probability Surveys, Vol. 2, pp. 213-244.

Cherny, A. (2002) "On the Uniqueness in Law and the Pathwise Uniqueness for Stochastic Differential Equations," Theory of Probability and Its Applications. Vol. 46, pp, 406-419.

Chistyakov, V. (2004) "Selections of Bounded Variations," Journal of Applied Analysis, Vol. 10, No. 1, pp. $1-82$.

Crandall, M., Lions, P.-L. (1983) "Viscosity Solutions of Hamilton-Jacobi Equations," Transactions of the American Mathematical Society, Vol. 277, No. 1, pp. 1-42.

Dayanik, S., Karatzas, I. (2003) "On the Optimal Stopping Problem for One-Dimensional Diffusions," Stochastic Processes and their Applications, Vol. 107, No. 2, pp. 173-212.

Décamps, J.-P., Mariotti, T., Villeneuve, S. (2006) "Irreversible Investment in Alternative Projects" Economic Theory, Vol. 28, pp. 435-448.

De Coster, C., Habets, P. (2006) Two-Point Boundary Value Problems: Lower and Upper Solutions, Elsevier Science.

Demarzo, P., Fishman, M., He, Z., And Wang, N. (2012) "Dynamic Agency and the $q$ Theory of Investment," forthcoming in the Journal of Finance. 
DeMarzo, P., Sannikov, Y. (2006) "Optimal Security Design and Dynamic Capital Structure in a Continuous-Time Agency Model," Journal of Finance, Vol. 61, pp. 2681-2724.

Dixit, A. (1993) The Art of Smooth Pasting, Academic Press, Harwood.

Dixit, A. (1993) "Choosing Among Alternative Discrete Investment Projects under Uncertainty," Economics Letters, Vol. 41, pp. 265-268.

Duffie, D. (2001) Dynamic Asset Pricing Theory, Princeton University Press.

Dumas, B. (1991) "Super Contact and Related Optimality Conditions," Journal of Economic Dynamics and Control, Vol. 15, pp. 675-685.

Evans, L. C. (1983) "Classical Solutions of the Hamilton-Jacobi-Bellmann Equation of Uniformly Elliptic Operators," Transactions of the American Mathematical Society, Vol. 275, No. 1, pp. 245-255.

Fleming, W., Soner, H. (1993) Controlled Markov Processes and Viscosity Solutions, Springer Verlag, Springer.

Gilbarg, D., Trudinger, N. (2001) Elliptic Partial Differential Equations of Second Order, Springer Verlag, Springer.

Gittins, J. (1979) "Bandit Processes and Dynamic Allocation Indices," Journal of the Royal Statistical Society, Vol. 41, No. 2, pp. 148-177.

Harrison, M., Taksar, M. (1983) "Instantaneous Control of Brownian Motion," Mathematics of Operations Research, Vol. 8, No. 3, pp. 439-453.

Hartman, P. (1960) "On Boundary Value Problems for Systems of Ordinary, Nonlinear, Second Order Differential Equations," Transactions of the American Mathematical Society, Vol. 96, No. 3, pp. 493-509.

Hartman, P. (2002) Ordinary Differential Equations, Second Edition, Classics in Applied Mathematics, SIAM.

Josephy, M. (1981) "Composing Functions of Bounded Variation," Proceedings of the American Mathematical Society, Vol. 83, pp. 354-356.

Jovanovic, B. (1979) "Job Matching and the Theory of Turnover," Journal of Political Economy, Vol. 87(5), pp. 972-990 pp. 439-453.

Karatzas, I., Shreve, S. (1998) Brownian Motion and Stochastic Calculus, Second Edition, Springer.

Karlin, S., Rubin, H. (1956) "The Theory of Decision Procedures for Distributions With Monotone Likelihood Ratio," Annals of Mathematical Statistics, Vol. 27, pp. 272-299.

Keller, G., Rady, S., And Cripps, M. (2005) "Strategic Experimentation with Exponential Bandits," Econometrica, Vol. 73, No. 1, pp. 39-68.

Kiguradze, I. T. (1967) "A-priori Estimates for the Derivatives of Bounded Functions Satisfying SecondOrder Differential Inequalities," Differentsialnye Uravneniya, Vol. 3, pp. 1043-1052. 
Krylov, N. (1980) Controlled Diffusion Processes, Springer Verlag, Springer.

Lehmann, E. (1988) "Comparing Location Experiments," Annals of Statistics, Vol. 16, pp. 521-533.

Leland, H. (1994) "Corporate Debt Value, Bond Covenants, and Optimal Capital Structure," Journal of Finance, Vol. 49, pp. 1213-1252.

Lions, P.-L. (1983) "Optimal Control of Diffusion Processes and Hamilton-Jacobi-Bellman Equations," Communications in Partial Differential Equations, Vol. 8, No. 11, pp. 1229-1276.

McDonald, R., Siegel, D. (1986) "The Value of Waiting to Invest," Quarterly Journal of Economics, Vol. 101(4), pp. 707-727.

Merton, R. (1969) "Lifetime Portfolio Selection under Uncertainty: the Continuous Time Case," Review of Economic and Statistics, Vol. 51, pp. 247-257.

Merton, R. (1971) "Optimum Consumption and Portfolio Rules in a Continous Time Model," Journal of Economic Theory, Vol. 3, pp. 373-413.

Milgrom, P., Shannon, C. (1994) "Monotone Comparative Statics," Econometrica, Vol. 62, pp. 157-180.

Milgrom, P., Segal, I. (2002) "Envelope Theorems for Arbitrary Choice Sets," Econometrica, Vol. 70(2), pp. 583-601.

Moscarini, G., Smith, L. (2001) "The Optimal Level of Experimentation," Econometrica, Vol. 69(6), pp. $1629-1644$.

NaKaO, S. (1972) "On the Pathwise Uniqueness of Solutions of One-Dimensional Stochastic Differential Equations," Osaka Journal of Mathematics, Vol. 9, pp. 513-518.

Noussair, E. S. (1979) "On the Existence of Solutions to Nonlinear Elliptic Boundary Value Problems," Journal of Differential Equations, Vol. 34, No. 3, pp. 482-495.

Ørsendal, B., Sulem, A. (2007) Applied Stochastic Control of Jump Diffusions, Second Edition, Springer.

Øksendal, B. (2007) Stochastic Differential Equations: An Introduction with Applications, Sixth Edition, Springer.

PešKir, G. And Shiryaev, A. (2006) Optimal Stopping and Free-Boundary Problems, Birkhauser Boston.

Pham, H. (2009) Continuous-time Stochastic Control and Optimization with Financial Applications, Springer Verlag.

Quah, J., Strulovici, B. (2009) "Comparative Statics, Informativeness, and the Interval Dominance Order," Econometrica, Vol. 77, pp. 1949-1992.

Quah, J., Strulovici, B. (2012) "Aggregating the Single Crossing Property," Econometrica, Vol. 80, pp. 2333-2348.

Quah, J., Strulovici, B. (2013) "Discounting, Values, and Decisions," Journal of Politicial Economy, Vol. 121, pp. 896-939. 
Revuz, D., Yor, M. (2001) Continuous Martingales and Brownian motion, Third Edition, Springer Verlag.

Rincón-Zapatero, J., Santos, M. (2009) "Differentiability of the value function without interiority assumptions," Journal of Economic Theory, Vol. 144(5), pp. 1948-1964.

Rincón-Zapatero, J., Santos, M. (2010) "Differentiability of the Value Function in Continuous-Time Economic Models," Working Paper.

SAfonov, M. V. (1989) "On the Classical Solution of Nonlinear Elliptic Equations of Second Order," Mathematics of the USSR-Izvestiya, Vol. 33, No. 3, pp. 597-612.

Sannikov, Y. (2008) "A Continuous-Time Version of the Principal-Agent Problem," Review of Economic Studies, Vol. 75(3), pp. 957-984.

Schrader, K. (1969) "Existence theorems for second order boundary value problems," Journal of Differential Equations, Vol. 5, pp. 572-584.

Shreve, S. E., Lehoczky, J. P., Gaver, D. P. (1984) "Optimal Consumption for General Diffusions with Absorbing and Reflecting Barriers," SIAM Journal on Control and Optimization, Vol. 22, No. 1, pp. 55-75.

Stroock, D., Varadhan, S. (1979) Multidimensional Diffusion Processes, Springer.

Szydlowski, M. (2014) "Incentives, Project Choice and Dynamic Multitasking," Working Paper.

Topkis, D. (1978) "Minimizing a Submodular Function on a Lattice," Operations Research, Vol. 26, pp. 305321.

Touzi, N. (2010) Deterministic and Stochastic Control, Application to Finance, Lecture Notes, Départment de Mathematiques Appliques, Ecole Polytechnique.

Veinote, A. (1989) "Lattice Programming," Unpublished Lecture Notes, Stanford University.

Veretennikov, A. (1981) "On Strong Solutions and Explicit Formulas for Solutions of Stochastic Intergal Equations," Math USSR Sb., Vol. 39, pp. 387-403.

Villeneuve, S., (2007) "On Threshold Strategies and the Smooth-Fit Principle for Optimal Stopping Problems," Journal of Applied Probability, Vol. 44, No. 1, pp. 181-198.

Yamada, T., Watanabe, S. (1971) "On the Uniqueness of Solutions of Stochastic Differential Equations," Journal of Mathematics of Kyoto University, Vol. 11, pp. 155-167.

Yong, J., Zhou, X.-Y. (1999) Stochastic Controls, Springer Verlag. 